\title{
Microbiomes of biohydrogen production from dark fermentation of industrial wastes: current trends, advanced tools and future outlook
}

\author{
Eka Latiffah Nadia Dzulkarnain ${ }^{1}$, Jemilatu Omuwa Audu ${ }^{1,2}$, Wan Rosmiza Zana Wan Dagang ${ }^{1}$ and \\ Mohd Firdaus Abdul-Wahab ${ }^{1,3^{*}}$ (1)
}

\begin{abstract}
Biohydrogen production through dark fermentation is very attractive as a solution to help mitigate the effects of climate change, via cleaner bioenergy production. Dark fermentation is a process where organic substrates are converted into bioenergy, driven by a complex community of microorganisms of different functional guilds. Understanding of the microbiomes underpinning the fermentation of organic matter and conversion to hydrogen, and the interactions among various distinct trophic groups during the process, is critical in order to assist in the process optimisations. Research in biohydrogen production via dark fermentation is currently advancing rapidly, and various microbiology and molecular biology tools have been used to investigate the microbiomes. We reviewed here the different systems used and the production capacity, together with the diversity of the microbiomes used in the dark fermentation of industrial wastes, with a special emphasis on palm oil mill effluent (POME). The current challenges associated with biohydrogen production were also included. Then, we summarised and discussed the different molecular biology tools employed to investigate the intricacy of the microbial ecology associated with biohydrogen production. Finally, we included a section on the future outlook of how microbiome-based technologies and knowledge can be used effectively in biohydrogen production systems, in order to maximise the production output.
\end{abstract}

Keywords: Biohydrogen microbiomes, Biohydrogen production, Dark fermentation, Palm oil mill effluent, Industrial wastes, Molecular biology tools

\section{Introduction}

Dark fermentation is a biological decomposition process reported to be one of the most promising approaches for the treatment of organic wastes. This is also the process commonly used in sustainable bioenergy production. A recent study by the World Bank in 2018 predicted that the global waste production will grow to 3.4 billion tonnes by 2050 , with organic wastes generated from agricultural

\footnotetext{
*Correspondence: firdausw@utm.my

${ }^{1}$ Department of Biosciences, Faculty of Science, Universiti Teknologi

Malaysia, 81310 Skudai, Johor, Malaysia

Full list of author information is available at the end of the article
}

sectors representing more than $50 \%$ of the total waste composition (Kaza et al. 2018). This large amount of wastes has to be sustainably managed. For this purpose, dark fermentation can offer two simultaneous benefits of both waste treatment and sustainable bioenergy generation (Wang and Yin 2019). Methane is currently the commonly produced bioenergy from organic wastes, but hydrogen production is also gaining attention, as part of the hydrogen economy, to substitute the hydrogen produced from fossil fuels. Hydrogen has three times higher energy content $(120 \mathrm{MJ} / \mathrm{kg})$ than hydrocarbon fuels, and its combustion is clean and carbon free, producing only water as the by-product (Zhang et al. 2020). Dark 
fermentation is more attractive than the other biological processes, due to the low demand for light (unlike the photosynthetic routes), capable of high biohydrogen production rate, environmentally friendly, versatile substrate utilisation and less energy intensive (Ghimire et al. 2015; Mishra et al. 2019). In addition, the use of organic wastes as feedstocks in dark fermentative biohydrogen production is potentially cost competitive, since organic wastes are relatively abundant, renewable, cheap and highly biodegradable (Sharma et al. 2020).

Various renewable organic wastes such as sake lees, cassava, sago, glycerol, rice straw, vegetable waste, food waste, date seeds, sugarcane molasses, corn stover, alligator weed, oil palm sap and wheat straw have been explored as the potential substrate for dark fermentative biohydrogen production (Chen et al. 2021; Choiron et al. 2020; Li et al. 2020; Liu et al. 2013; Moreno-Andrade et al. 2015; Noparat et al. 2012; Oliveira et al. 2020; Panin et al. 2020; Pason et al. 2020; Rambabu et al. 2020; Saleem et al. 2020; Ulhiza et al. 2018; Zhang et al. 2011). Palm oil mill effluent (POME), a wastewater generated in large quantity during palm oil extraction process is another renewable organic waste of interest that is currently under intense investigations as biohydrogen production substrate (Abdullah et al. 2020; Akhbari et al. 2021; Audu et al. 2021; Jamali et al. 2019; Zainal et al. 2018). The use of both pure, as well as mixed culture as the inoculum in the dark fermentation reactor have been investigated. A mixed culture system is generally more preferable and practical over pure culture system, due to the diverse microbial communities present that can rapidly degrade a wide range of substrates. A strict aseptic condition is also not required, making its handling easier with cheaper cost of operation (Nitipan et al. 2014; Pachapur et al. 2019). Nonetheless, the co-existence of biohydrogen producers with non-biohydrogen producers, and biohydrogen-consumers such as methanogens and homoacetogens in the mixed culture, makes it a very biochemically complex environment. Despite the multiple studies carried out, there is still a gap in the understanding of the biological mechanisms of dark fermentation for biohydrogen production, including the specific microbial community and the trophic interactions (Cabrol et al. 2017; Das 2017). The methane-producing fermentation systems are more well characterised in this aspect.

Microbiomes are classically defined as the community consisting of microorganisms with distinct properties and metabolic functions, interacting with its environment which results in the formation of specific ecological niche (Whipps et al. 1988). The term "microbiome" was often used interchangeably with "microbiota", but recently there has been efforts to distinguish these two. Berg et al. (2020) defined "microbiota" as the assemblage of living microorganisms (i.e. the bacteria, archaea, fungi, microalgae and the protists, excluding phages, viruses, plasmids, prions, viroids, and free DNA), while the "microbiomes" are the microbiota and their structural elements, metabolites/signal molecules, and the surrounding environmental conditions (Berg et al. 2020). Phages, viruses, plasmids, prions, viroids, and free DNA are part of the microbiomes. This review will refer to this updated definition.

Taxonomic classification of biogas microbiomes is often accomplished using sequence similarity searches against $16 \mathrm{~S}$ ribosomal RNA (rRNA) gene reference databases, such as SILVA (Akhbari et al. 2021), Greengenes (Oliveira et al. 2020), Ribosomal Database Project (RDP) (Cho et al. 2018) or National Center for Biotechnology Information (NCBI) (Mazareli et al. 2020). However, the genome sequences of biogas-producing microorganisms are underrepresented in these reference databases, which hinder the reliable taxonomic classification for microbiomes present in the biogas production systems (Hassa et al. 2018). Functional roles of biogas microbiome are often inferred to related species in public genome database based on the 16S rRNA gene sequence similarity (Campanaro et al. 2016). Therefore, it is imperative to have a comprehensive reference database to improve the taxonomic annotation of biogas-producing microbiomes across the entire microbial databases. Metagenomics has been used in many biogas-producing studies to decipher the taxonomic diversity, metabolic functions and the physiology of biogas-producing microbiomes. This has led to the compilation of metagenome-assembled genomes (MAGs) belonging to the biogas-producing microbiomes in a repository, called the "Biogasmicrobiome" (https://biogasmicrobiome.env.dtu.dk/) (Campanaro et al. 2020). This database contains a collection of 1600 MAGs of bacterial and archaeal species that underpin various biogas production systems, substantially expanding the existing public genome databases (Campanaro et al. 2020). In addition, Microbial Database for Activated Sludge (MiDAS) Field Guide (https:// www.midasfieldguide.org/guide/search) is an ecosystem-specific database for wastewater treatment systems which aims to facilitate collaborative research and compile information on the physiology and ecology of the key microorganisms present in activated sludge wastewater treatment systems (Mcllroy et al. 2015). MiDAS 4 offers a comprehensive set of full-length amplicon sequence variant (ASV)-resolved 16S rRNA gene sequences which covers over 31,000 species, allowing researchers to dig into the microbiome compositions of activated sludge, anaerobic digesters and wastewater treatment systems at the genus to species level resolutions (Dueholm et al. 2021). 
Dark fermentation for biohydrogen production is mediated by many different groups of microorganisms, to convert complex organic wastes into biohydrogen, volatile fatty acids and carbon dioxide $\left(\mathrm{CO}_{2}\right)$ (Hay et al. 2013). The efficiency and stability of dark fermentation system relies on the syntrophic activity of the microbial community belonging to different functional guilds, working in tight interaction (Cabrol et al. 2017). It has been reported that the understanding of the species composition, specific metabolic functions, and interspecies interactions are often more important than the species richness itself in maintaining the overall performance of the system (Cabrol et al. 2017). The rapid advancement of molecular tools has contributed to the major discoveries of the diversity and structure of the biohydrogen-producing consortia. In a mixed culture system, the microbiomes involved are phylogenetically diverse, with multiple contributions in the biohydrogen production and the breakdown of organic wastes (Cabrol et al. 2017).

This review summarises and evaluates the distinct microbial communities present in a biohydrogen production systems, and the molecular tools that have been used for microbiome analysis in biohydrogen production from industrial wastewater and POME. We also included a future outlook of how microbiome-based technologies and knowledge can be used effectively in biohydrogen production systems, in order to maximise the production output.

\section{Microbiomes in dark fermentative biohydrogen production}

The microorganisms present in dark fermentative biohydrogen production system include both the biohydrogen producers and non-producers. Biohydrogen producers possess the ability to convert complex organic substrates into biohydrogen in the absence of light. They can exist as a single strain or a community of various taxa. They can be found in a diverse environment, such as POME sludge (Jamali et al. 2019; Mahmod et al. 2019; Zainal et al. 2018), sludge from municipal wastewater plants (Chang et al. 2011; Viana et al. 2019), sludge from food waste (Li et al. 2018), cattle dung (Sen and Suttar 2012), pig manure (Wang et al. 2011) and marine sediments (Liu et al. 2018), many of which has been extensively studied. In general, Clostridium (Gram positive, spore former) and Enterobacter (Gram negative, non-spore former) are the most common biohydrogen-producing genera reported, for mesophilic conditions (Kumar et al. 2018). While under thermophilic and hyperthermophilic conditions, Clostridium, Thermoanaerobacterium, Thermotoga and Caldicellulosiruptor dominate (O-Thong 2017). Research on biohydrogen production using the lower temperature-adapted psychrophiles and psychrotrophs are still somewhat limited (Alvarado-Cuevas et al. 2015; Mohammed et al. 2018). In addition, other genera including Bacillus, Ethanoligenens, Klebsiella, Citrobacter and Escherichia also frequently reported as the biohydrogen producers. Non-biohydrogen producers on the other hand, could interfere with the overall biohydrogen yield, by either consuming the hydrogen produced, competing with the biohydrogen producers for substrates, or inhibit biohydrogen producers with their produced metabolites which eventually decrease the efficiency of the biohydrogen production system as a whole (Cabrol et al. 2017). Inoculum pre-treatment has become necessary in mixed culture systems in order to selectively enrich the biohydrogen producers and inactivate the hydrogen consumers.

The main biochemical pathways in dark fermentation overlaps with those of anaerobic digestion, where diverse microbial communities synergistically work together to ensure a stable degradation of organic substrates (Abendroth et al. 2015; Stolze et al. 2016). The pathways can be divided into four phases: hydrolysis, acidogenesis, acetogenesis, and methanogenesis (Fig. 1). In anaerobic digestion, hydrogen $\left(\mathrm{H}_{2}\right)$ is produced during acidogenesis and acetogenesis, by hydrolytic and fermentative bacteria. It is later consumed during methanogenesis, when methanogenic archaea use $\mathrm{H}_{2}$ and $\mathrm{CO}_{2}$ to produce methane $\left(\mathrm{CH}_{4}\right)$ (Hassa et al. 2018). Therefore, inhibition of methanogenesis is necessary to re-direct the pathway for hydrogen production, through the final step of dark fermentation. The initial hydrolysis starts when the complex substrates (polysaccharides, lipids, and proteins) are hydrolysed to monomers (sugars, amino acids, fatty acids) by the actions of extracellular hydrolytic enzymes such as cellulase, pectinase, lipase and protease. The microbial taxa responsible for the hydrolytic activities are mainly represented by two phyla, Firmicutes and Bacteroidetes, from the genera Clostridium and Bacteroides. The abundance of these phyla can be attributed to their degradative abilities, as the main degraders of cellulolytic materials (Abendroth et al. 2015). Members of these phyla are also known to be fast growers, utilising the hydrolysed products for growth through fermentation, and are usually present in the whole degradation process. They are also less sensitive to changes in environmental conditions (Li et al. 2017; Wang et al. 2018). The hydrolysis step is usually not a rate-limiting step, except with recalcitrant substrates such as lignocellulosic waste, which usually results in incomplete hydrolysis requiring a pre-treatment step (Menzel et al. 2020).

Next, in acidogenesis, the hydrolysed products are further metabolised to $\mathrm{H}_{2}, \mathrm{CO}_{2}$, and short-chain fatty acids (SCFA) (e.g. acetate, butyrate, formate, propionate, etc.) by acidogenic microbial communities. The predominant 


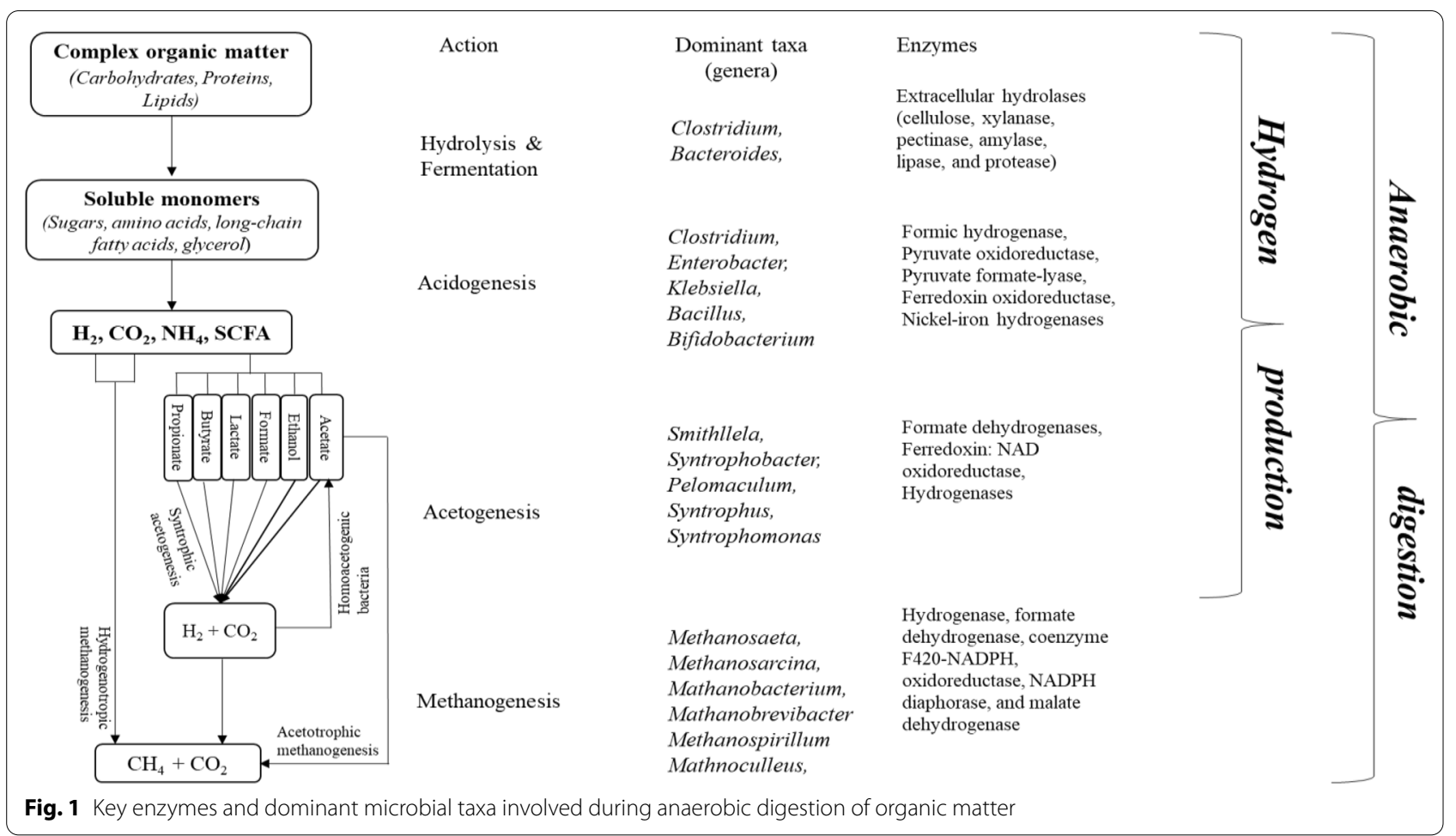

phyla associated with this phase are Bacteroidetes, Firmicutes, Chloroflexi, and Proteobacteria (Audu et al. 2021; Castellano-Hinojosa et al. 2018). Acidogenesis is usually a rapid process, accompanied by the accumulation of SCFA and the subsequent drop in $\mathrm{pH}$. The microorganisms in the acidogenic phase consist of both facultative and obligate anaerobes and are often referred to as acidogens, or acid formers. The commonly reported genera participating in this phase are Clostridium, Bacteroides, Bifidobacterium, Bacillus, and Streptococcus (GonzalezMartinez et al. 2016; Seon et al. 2014). In this phase, carbohydrates (mostly glucose, the preferred substrates) are converted to pyruvate through the glycolytic pathway (Saravanan et al. 2021; Vardar-Schara et al. 2008). Under mesophilic condition, the $\mathrm{H}_{2}$-yielding fermentation routes are the obligate anaerobic (Clostridium type) and facultative anaerobic (Enterobacteria type) fermentation route.

In the facultative anaerobic fermentation route, pyruvate is further converted to acetyl-CoA and formate, by pyruvate formate lyase (PFL), and $\mathrm{H}_{2}$ is produced from formate by the formate hydrogen lyases enzyme complex. The strict anaerobic fermentation route involves the oxidisation of pyruvate to acetyl-CoA and reduced ferredoxin ( $\mathrm{Fd}$ ) by pyruvate ferredoxin oxidoreductase (PFOR). $\mathrm{H}_{2}$ is then released from the reduced $\mathrm{Fd}$ by the action of hydrogenase. Additional molecules of $\mathrm{H}_{2}$ can also be produced from the oxidisation of nicotinamide adenine dinucleotide (NADH) to reduced Fd by NADH:ferredoxin oxidoreductase (NFOR), followed by the subsequent release of $\mathrm{H}_{2}$ from the reduced Fd by hydrogenase (Fig. 2). However, the activities of NFOR is usually inhibited under standard conditions and can only proceed when $\mathrm{H}_{2}$ partial pressure is very low, as opposed to PFOR which is still active at standard $\mathrm{H}_{2}$ partial pressure (Kraemer and Bagley 2007). In addition to $\mathrm{H}_{2}$, acetyl-CoA can also be further converted to non-gaseous products including SCFA (acetate, lactate, butyrate, propionate), alcohols (ethanol, butanol), and ketones (acetone). The overall theoretical maximum yield of $\mathrm{H}_{2}$ from the reduced Fd pathway is $4 \mathrm{~mol}$ of $\mathrm{H}_{2}$ per $1 \mathrm{~mol}$ of glucose, and $2 \mathrm{~mol}$ of $\mathrm{H}_{2}$ per $1 \mathrm{~mol}$ of glucose from the formate pathway. The yield is influenced by the fermentation end products generated alongside $\mathrm{H}_{2}$. Theoretically, based on the 'Thauer limit', the maximum yield of $4 \mathrm{~mol}$ of $\mathrm{H}_{2}$ can be obtained with acetate as the fermentation end product, $2 \mathrm{~mol}$ with butyrate or propionate, and much lower yields when alcohols are the end products (Keskin et al. 2019; Vardar-Schara et al. 2008).

The intermediates products $\mathrm{H}_{2}, \mathrm{CO}_{2}$, and acetate can directly be utilised by methanogens for methane production, while other products such as butyrate, propionate, valerate require further transformation or conversion through syntrophic acetogenesis first (Lim et al. 2020). In the acetogenesis phase, unusable substrates are converted to acetate, $\mathrm{CO}_{2}$, and $\mathrm{H}_{2}$ 


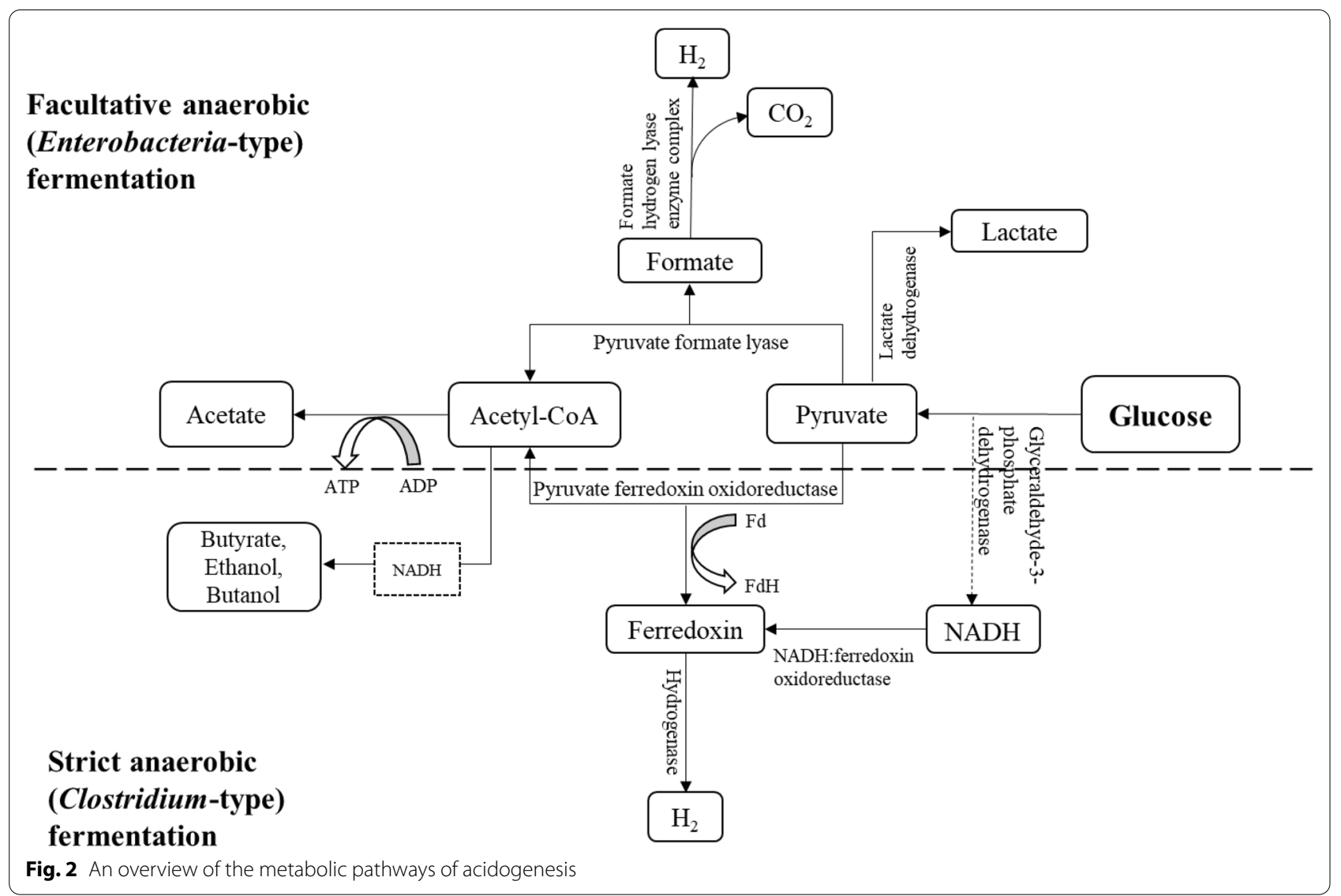

by hydrolytic and fermentative bacteria that do not possess hydrolytic activities. Acetogenesis is also the rate-limiting phase. In addition, the $\mathrm{H}_{2}$ produced from acetogenesis is converted to $\mathrm{CH}_{4}$ by the hydrogenotrophic methanogens (Venkiteshwaran et al. 2015). The oxidation of non-gaseous products of acidogenesis is based on the reverse electron transfer process, a thermodynamically unfavourable condition. The process requires energy input to drive the oxidation/reduction process involving multiple enzyme systems, such as formate dehydrogenases, ferredoxin:NAD oxidoreductases, hydrogenases, reactive quinone complexes, $c$-type cytochromes, etc. (Sieber et al. 2012). However, when oxidation is coupled with methane production, energy conversion is more feasible due to the diminishing effects of $\mathrm{H}_{2}$ pressure created by the methanogenic activity (Sikora et al. 2017). The most commonly reported syntrophic acetogens in anaerobic digester are the propionate degraders belonging to the genera Pelotomaculum, Smithllela, and Syntrophobacter. While the oxidation of butyrate and other fatty acids is carried out by Syntrophus and Syntrophomonas (Venkiteshwaran et al. 2015). The acetogenesis phase is important because it ensures rapid and stable anaerobic digester operation by preventing methanogenic inhibition due to the high acid concentrations (Wang et al. 2018).

Methanogenesis is the final phase in anaerobic digestion, in which acetate, $\mathrm{H}_{2}$ and $\mathrm{CO}_{2}$ produced from the acidogenic and acetogenic phases are further transformed into biogas, in the form of $\mathrm{CH}_{4}$ and $\mathrm{CO}_{2}$. Methanogens are the main hydrogen consumers in the anaerobic environments, and for this reason they are usually suppressed in biohydrogen dark fermentation to maximise the hydrogen yield. Unlike the previous three phases which are dominated by fermentative bacteria, the methanogenesis phase is exclusively dominated by the methanogenic archaea. The methanogens are slow growers and sensitive to environmental changes. Methanogenesis can occur via three possible pathways based on the available substrate: acetoclastic, methylotrophic, or hydrogenotrophic methanogenesis. Acetotrophic/acetoclastic methanogens use acetate as substrate by catalysing its methane production, methylotrophic methanogens use methyl-based compounds, and hydrogenotrophic methanogens use $\mathrm{CO}_{2}$ and $\mathrm{H}_{2}$ for $\mathrm{CH}_{4}$ production (Hassa et al. 2018; Lim et al. 2020; Menzel et al. 2020). The commonly observed methanogens associated with biogas production are from the genera Methanosaeta and Methanosarcina (acetotrophic 
methanogens); and Methanobacterium, Methanospirillum, Methanococcus, and Methanobrevibacter (hydrogenotrophic methanogens) (Castellano-Hinojosa et al. 2018). The acetotrophic methanogens have been reported to be the most predominant type of methanogen in anaerobic digesters, and are responsible for about $70 \%$ of the methane generated (Gonzalez-Martinez et al. 2016). The genus Methanosaeta for example, are obligate acetoclastic methanogens, characterised as slow growers, and only use acetate as the substrate. While Methanosarcina are facultative acetoclastic methanogens, have faster growth rate, and can utilise a wider substrate range in addition to acetate. Unlike Methanosaeta, members of the Methanosarcina genus have a low affinity to acetate, which can account for its abundance in high acetate concentration condition by outgrowing the Methanosaeta population. At low acetate concentration, Methanosaeta have been reported to dominate the archaea community. Due to their high affinity to acetate, Methanosaeta genus outcompete Methanosarcina population by utilising the available acetate in the environment (Conklin et al. 2006; Lim et al. 2020).

The intricacy and complexity of dark fermentation makes it a process "black box", due to the variety of multiple metabolic activities and interactions within the microbial community, along with the limited biogas-producing microbial genomes in the reference databases. So far, biohydrogen yield obtained in practice are mostly up to $32 \%$, hampered by the Thauer limit (Patel et al. 2018). In addition, biohydrogen production from dark fermentation of organic wastes seldom exceeded $2 \mathrm{~mol} \mathrm{H}_{2} / \mathrm{mol}$ hexose (Wang and Yin 2019). A number of approaches have been explored to overcome the bottleneck of dark fermentative biohydrogen production, including reactor configurations, operational condition, inoculum and substrate types, pre-treatment strategies and integrating multiple biohydrogen production systems (Audu et al. 2020). The microbiomes of the systems are the integral part of these processes. Identifying the microorganisms and understanding the behaviour is crucial to dark fermentation robustness, as elaborated in sections "Industrial wastes as substrates" and "Palm oil mill effluent (POME) as substrate".

\section{Industrial wastes as substrates}

Dark fermentative biohydrogen production has been investigated using a diverse range of pure cultures (Table 1). Clostridium butyricum represents the most commonly studied pure culture under mesophilic condition. C. butyricum is known for its high biohydrogen yield regardless of the substrates complexity, which can range from simple carbohydrates such as glucose, xylose and sucrose, to complex biomass such as food waste (Kanchanasuta et al. 2017), glycerol (Kivistö et al. 2013; Yin and Wang 2017), microalgae (Ortigueira et al. 2015) and sugarcane bagasse (Plangklang et al. 2012). At present, the highest reported biohydrogen yield from the conversion of organic waste by $C$. butyricum was $3.0 \mathrm{~mol} \mathrm{H}_{2} / \mathrm{mol}$ glycerol which is equal to $3.6 \mathrm{~mol}$ $\mathrm{H}_{2} /$ mol hexose out of the theoretical stoichiometric yield ( $4 \mathrm{~mol} \mathrm{H}_{2} / \mathrm{mol}$ hexose, the Thauer limit) when fermenting raw glycerol from biodiesel production process (Kivistö et al. 2013). In addition, other Clostridia species including C. beijerinckii (Rambabu et al. 2021), C. pasteurianum (Sarma et al. 2019), C. acetobutylicum (Azman et al. 2016) and C. saccharoperbutylacetonicum (Dada et al. 2013) have also been investigated for biohydrogen production from organic wastes under mesophilic condition. Under thermophilic conditions, C. thermocellum has been reported to be the ideal strain. Rambabu et al. (2020) obtained $103.97 \mathrm{mmol}$ $\mathrm{H}_{2} / \mathrm{L}$ using C. thermocellum to produce biohydrogen from date seeds waste through dark fermentation system operated at $50{ }^{\circ} \mathrm{C}$ and initial $\mathrm{pH} 7$. Tian et al. (2015) also used C. thermocellum to ferment sugarcane bagasse at $55{ }^{\circ} \mathrm{C}$ and obtained $4.89 \mathrm{mmol} \mathrm{H}_{2} /$ g medium added. Versatile substrate utilisation with high biohydrogen yields of $0.23-3.47 \mathrm{H}_{2} / \mathrm{mol}$ hexose from $C$. butyricum and $0.52-3.0 \mathrm{~mol} \mathrm{H}_{2} / \mathrm{mol}$ hexose from the other Clostridium species have made Clostridia popular for use in dark fermentation (Wang and Yin 2019). However, the strict anaerobic requirement of Clostridia complicates their practical applications.

Facultative anaerobes such as Enterobacter, Klebsiella, Citrobacter, Escherichia and Bacillus are the alternative candidates for biohydrogen dark fermentation. These species possess the ability to shift from aerobic respiration producing adenosine triphosphate (ATP) in the presence of oxygen, to fermentation in the absence of oxygen (Lukajtis et al. 2018). Nevertheless, facultative anaerobes generally produce lower biohydrogen yield than the strict anaerobes, e.g. Clostridia, but the high tolerance to oxygen makes them more attractive for practical applications. Pure cultures have commonly been used for investigations on biohydrogen production and the related metabolic activity. This allows the investigation into the metabolic pathways involved, and subsequently the feasible ways to enhance the biohydrogen production efficiency through metabolic engineering (Wang and Yin 2019). In addition, reliable biohydrogen yields by avoiding the production of undesired by-products, reproducible bioprocess and ease of genetic manipulations are the other benefits of using pure cultures (Kumar et al. 2018). However, aseptic condition is compulsory to handle pure cultures as they are highly susceptible to contaminations. They are also substrate selective, and developing pure 
Table 1 Dark fermentative biohydrogen production from various substrates (including industrial wastes) using pure culture

\begin{tabular}{|c|c|c|c|c|c|c|}
\hline \multirow[t]{2}{*}{ Microorganism } & \multirow[t]{2}{*}{ Substrate } & \multirow[t]{2}{*}{ Reactor type } & \multicolumn{2}{|c|}{ Operating conditions } & \multirow[t]{2}{*}{ Biohydrogen yield } & \multirow[t]{2}{*}{ References } \\
\hline & & & Temperature, $T$ & $\mathrm{pH}$ & & \\
\hline $\begin{array}{l}\text { Clostridium butyri- } \\
\text { cum INET } 1\end{array}$ & Glucose & Batch & $35^{\circ} \mathrm{C}$ & $\begin{array}{l}\text { Initial=7.0, } \\
\text { Operation= uncon- } \\
\text { trolled }\end{array}$ & $\begin{array}{l}2.24 \mathrm{~mol} \mathrm{H}_{2} / \mathrm{mol} \\
\text { hexose }\end{array}$ & Yin and Wang (2017) \\
\hline $\begin{array}{l}\text { Clostridium butyri- } \\
\text { cum INET } 1\end{array}$ & Xylose & Batch & $35^{\circ} \mathrm{C}$ & $\begin{array}{l}\text { Initial=7.0, } \\
\text { Operation = uncon- } \\
\text { trolled }\end{array}$ & $\begin{array}{l}1.23 \mathrm{~mol} \mathrm{H}_{2} / \mathrm{mol} \\
\text { hexose }\end{array}$ & Yin and Wang (2017) \\
\hline $\begin{array}{l}\text { Clostridium butyri- } \\
\text { cum INET } 1\end{array}$ & Sucrose & Batch & $35^{\circ} \mathrm{C}$ & $\begin{array}{l}\text { Initial=7.0, } \\
\text { Operation = uncon- } \\
\text { trolled }\end{array}$ & $\begin{array}{l}1.44 \mathrm{~mol} \mathrm{H}_{2} / \mathrm{mol} \\
\text { hexose }\end{array}$ & Yin and Wang (2017) \\
\hline $\begin{array}{l}\text { Clostridium butyri- } \\
\text { cum INET } 1\end{array}$ & Lactose & Batch & $35^{\circ} \mathrm{C}$ & $\begin{array}{l}\text { Initial=7.0, } \\
\text { Operation = uncon- } \\
\text { trolled }\end{array}$ & $\begin{array}{l}1.83 \mathrm{~mol} \mathrm{H}_{2} / \mathrm{mol} \\
\text { hexose }\end{array}$ & Yin and Wang (2017) \\
\hline $\begin{array}{l}\text { Clostridium butyri- } \\
\text { cum INET } 1\end{array}$ & Starch & Batch & $35^{\circ} \mathrm{C}$ & $\begin{array}{l}\text { Initial=7.0, } \\
\text { Operation = uncon- } \\
\text { trolled }\end{array}$ & $\begin{array}{l}2.17 \mathrm{~mol} \mathrm{H}_{2} / \mathrm{mol} \\
\text { hexose }\end{array}$ & Yin and Wang (2017) \\
\hline $\begin{array}{l}\text { Clostridium butyri- } \\
\text { cum INET } 1\end{array}$ & Glycerol & Batch & $35^{\circ} \mathrm{C}$ & $\begin{array}{l}\text { Initial=7.0, } \\
\text { Operation = uncon- } \\
\text { trolled }\end{array}$ & $\begin{array}{l}0.67 \mathrm{~mol} \mathrm{H}_{2} / \mathrm{mol} \\
\text { hexose }\end{array}$ & Yin and Wang (2017) \\
\hline $\begin{array}{l}\text { Clostridium butyri- } \\
\text { cum TISTR } 1032\end{array}$ & Food waste & CSTR & $37^{\circ} \mathrm{C}$ & $\begin{array}{l}\text { Initial=6.0, } \\
\text { Operation = uncon- } \\
\text { trolled }\end{array}$ & $362 \mathrm{~mL} \mathrm{H}_{2} / \mathrm{g} \mathrm{VS}$ & $\begin{array}{l}\text { Kanchanasuta et al. } \\
(2017)\end{array}$ \\
\hline $\begin{array}{l}\text { Clostridium butyri- } \\
\text { cum DSM 10,702 }\end{array}$ & Microalgae & Batch & $37^{\circ} \mathrm{C}$ & ND & $\begin{array}{l}2.74 \mathrm{~mol} \mathrm{H}_{2} / \mathrm{mol} \\
\text { glucose }\end{array}$ & Ortigueira et al. (2015) \\
\hline $\begin{array}{l}\text { Clostridium butyri- } \\
\text { cum CWBI } 1009\end{array}$ & Glucose & TBSBR & $30^{\circ} \mathrm{C}$ & $\begin{array}{l}\text { Initial }=5.2, \\
\text { Operation }=5.2\end{array}$ & $\begin{array}{l}1.67 \mathrm{~mol} \mathrm{H}_{2} / \mathrm{mol} \\
\text { glucose }\end{array}$ & $\begin{array}{l}\text { Puhulwella et al. } \\
\text { (2014) }\end{array}$ \\
\hline $\begin{array}{l}\text { Clostridium butyri- } \\
\text { cum }\end{array}$ & Glycerol & Batch & $37^{\circ} \mathrm{C}$ & $\begin{array}{l}\text { Initial=7.4, } \\
\text { Operation = uncon- } \\
\text { trolled }\end{array}$ & $\begin{array}{l}3.0 \mathrm{~mol} \mathrm{H} \mathrm{H}_{2} / \mathrm{mol} \\
\text { glycerol }\end{array}$ & Kivistö et al. (2013) \\
\hline $\begin{array}{l}\text { Clostridium butyri- } \\
\text { cum CWBI } 1009\end{array}$ & Glucose & AnSBR & $30^{\circ} \mathrm{C}$ & $\begin{array}{l}\text { Initial=7.6, } \\
\text { Operation = uncon- } \\
\text { trolled }\end{array}$ & $\begin{array}{l}2.2 \mathrm{~mol} \mathrm{H}_{2} / \mathrm{mol} \\
\text { glucose }\end{array}$ & Beckers et al. (2013) \\
\hline $\begin{array}{l}\text { Clostridium butyri- } \\
\text { cum CWBI } 1009\end{array}$ & Glucose & $A B R$ & $30^{\circ} \mathrm{C}$ & $\begin{array}{l}\text { Initial=8.5, } \\
\text { Operation = uncon- } \\
\text { trolled }\end{array}$ & $\begin{array}{l}2.49 \mathrm{~mol} \mathrm{H}_{2} / \mathrm{mol} \\
\text { glucose }\end{array}$ & Laurent et al. (2012) \\
\hline $\begin{array}{l}\text { Clostridium butyri- } \\
\text { cum TISTR } 1032\end{array}$ & Sugarcane bagasse & Serum bottle & $37^{\circ} \mathrm{C}$ & $\begin{array}{l}\text { Initial }=6.5 \\
\text { Operation }=6.5\end{array}$ & $\begin{array}{l}1.52 \mathrm{~mol} \mathrm{H}_{2} / \mathrm{mol} \\
\text { hexose }_{\text {used }}\end{array}$ & $\begin{array}{l}\text { Plangklang et al. } \\
\text { (2012) }\end{array}$ \\
\hline $\begin{array}{l}\text { Clostridium beijer- } \\
\text { inckii DSM } 791\end{array}$ & Rice mill wastewater & Serum bottle & $37^{\circ} \mathrm{C}$ & $\begin{array}{l}\text { Initial=7.0, } \\
\text { Operation = uncon- } \\
\text { trolled }\end{array}$ & $214.9 \mathrm{~mL} \mathrm{H} / \mathrm{L}$ & Rambabu et al. (2021) \\
\hline $\begin{array}{l}\text { Clostridium beijer- } \\
\text { inckii PS-3 }\end{array}$ & Oil palm sap & Serum bottle & $30^{\circ} \mathrm{C}$ & $\begin{array}{l}\text { Initial=7.0, } \\
\text { Operation = uncon- } \\
\text { trolled }\end{array}$ & $\begin{array}{l}141 \mathrm{~mL} \mathrm{H} / \mathrm{g} \text { sub- } \\
\text { strate }\end{array}$ & Noparat et al. (2012) \\
\hline $\begin{array}{l}\text { Clostridium pasteuri- } \\
\text { anum DSM } 525\end{array}$ & Glycerol & Serum bottle & $37^{\circ} \mathrm{C}$ & $\begin{array}{l}\text { Initial }=7.0 \\
\text { Operation }=7.0\end{array}$ & $\begin{array}{l}1.10 \mathrm{~mol} \mathrm{H}_{2} / \mathrm{mol} \\
\text { glycerol }\end{array}$ & Sarma et al. (2019) \\
\hline $\begin{array}{l}\text { Clostridium pasteu- } \\
\text { rianum }\end{array}$ & Glucose & Serum bottle & $35^{\circ} \mathrm{C}$ & $\begin{array}{l}\text { Initial=7.0, } \\
\text { Operation = uncon- } \\
\text { trolled }\end{array}$ & $\begin{array}{l}2.2 \mathrm{~mol} \mathrm{H}_{2} / \mathrm{mol} \\
\text { xylose }\end{array}$ & Hsieh et al. (2016) \\
\hline Clostridium $\mathrm{BOH} 3$ & Fruit waste & Serum bottle & $37^{\circ} \mathrm{C}$ & $\begin{array}{l}\text { Initial=6.8, } \\
\text { Operation = uncon- } \\
\text { trolled }\end{array}$ & $\begin{array}{l}359.97 \mathrm{~mL} \mathrm{H} / \mathrm{g} \text { TS } \\
\text { utilised }\end{array}$ & Mahato et al. (2020) \\
\hline $\begin{array}{l}\text { Clostridium ther- } \\
\text { mocellum ATCC } \\
27,405\end{array}$ & Date seeds & Serum bottle & $50^{\circ} \mathrm{C}$ & $\begin{array}{l}\text { Initial=7.0, } \\
\text { Operation = uncon- } \\
\text { trolled }\end{array}$ & $103.97 \mathrm{mmol} \mathrm{H}_{2} / \mathrm{L}$ & Rambabu et al. (2020) \\
\hline $\begin{array}{l}\text { Clostridium acetobu- } \\
\text { tylicum YM1 }\end{array}$ & Rice bran & Batch & $34^{\circ} \mathrm{C}$ & $\begin{array}{l}\text { Initial = 5.5, } \\
\text { Operation = uncon- } \\
\text { trolled }\end{array}$ & $\begin{array}{l}117.24 \mathrm{~mL} \mathrm{H} \mathrm{H}_{2} / \mathrm{g} \\
\text { sugar }_{\text {consumed }}\end{array}$ & Azman et al. (2016) \\
\hline
\end{tabular}


Table 1 (continued)

\begin{tabular}{|c|c|c|c|c|c|c|}
\hline \multirow[t]{2}{*}{ Microorganism } & \multirow[t]{2}{*}{ Substrate } & \multirow[t]{2}{*}{ Reactor type } & \multicolumn{2}{|c|}{ Operating conditions } & \multirow[t]{2}{*}{ Biohydrogen yield } & \multirow[t]{2}{*}{ References } \\
\hline & & & Temperature, $T$ & $\mathrm{pH}$ & & \\
\hline $\begin{array}{l}\text { Clostridium ther- } \\
\text { mocellum ATCC } \\
27,405\end{array}$ & Sugarcane bagasse & Serum bottle & $55^{\circ} \mathrm{C}$ & $\begin{array}{l}\text { Initial =6.6, } \\
\text { Operation = uncon- } \\
\text { trolled }\end{array}$ & $\begin{array}{l}4.89 \mathrm{mmol}_{2} / \mathrm{g} \\
\text { medium added }\end{array}$ & Tian et al. (2015) \\
\hline $\begin{array}{l}\text { Clostridium saccha- } \\
\text { roperbutylacetoni- } \\
\text { cum N1-4 }\end{array}$ & Rice bran & Batch & $30^{\circ} \mathrm{C}$ & $\begin{array}{l}\text { Initial =6.0, } \\
\text { Operation = uncon- } \\
\text { trolled }\end{array}$ & $\begin{array}{l}3.37 \mathrm{~mol} \mathrm{H}_{2} / \mathrm{mol} \\
\text { sugar } \\
\text { consumed }\end{array}$ & Dada et al. (2013) \\
\hline $\begin{array}{l}\text { Clostridium tyrobu- } \\
\text { tyricum Fya } 102\end{array}$ & Glucose & CSTR & $35^{\circ} \mathrm{C}$ & $\begin{array}{l}\text { Initial }=6.0, \\
\text { Operation =6.0 }\end{array}$ & $\begin{array}{l}1.06 \mathrm{mmol} \mathrm{H}_{2} / \mathrm{mmol} \\
\text { glucose }\end{array}$ & Whang et al. (2011) \\
\hline $\begin{array}{l}\text { Enterobacter aero- } \\
\text { genes ZJU1 }\end{array}$ & Aquatic weed & Batch & $37^{\circ} \mathrm{C}$ & $\begin{array}{l}\text { Initial =6.0, } \\
\text { Operation = uncon- } \\
\text { trolled }\end{array}$ & $62.2 \mathrm{~mL} \mathrm{H} / \mathrm{g} \mathrm{VS}$ & Song et al. (2020) \\
\hline Enterobacter asburiae & Lactose & Batch & $25.6^{\circ} \mathrm{C}$ & $\begin{array}{l}\text { Initial = 7.2, } \\
\text { Operation = uncon- } \\
\text { trolled }\end{array}$ & $\begin{array}{l}1.19 \mathrm{~mol} \mathrm{H}_{2} / \mathrm{mol} \\
\text { lactose }\end{array}$ & $\begin{array}{l}\text { Alvarez-Guzmán et al. } \\
\text { (2020) }\end{array}$ \\
\hline $\begin{array}{l}\text { Enterobacter aero- } \\
\text { genes CDC 819-56 }\end{array}$ & Sago wastewater & Serum bottle & $31^{\circ} \mathrm{C}$ & $\begin{array}{l}\text { Initial =6.8, } \\
\text { Operation = uncon- } \\
\text { trolled }\end{array}$ & $\begin{array}{l}7.42 \mathrm{mmol} \mathrm{H}_{2} / \mathrm{mol} \\
\text { glucose }\end{array}$ & Ulhiza et al. (2018) \\
\hline $\begin{array}{l}\text { Enterobacter aero- } \\
\text { genes PTCC } 1221\end{array}$ & Rice straw & Serum bottle & $37^{\circ} \mathrm{C}$ & $\begin{array}{l}\text { Initial }=5.8 \\
\text { Operation }=5.8\end{array}$ & 19.73 mL H $H_{2} / g$ straw & $\begin{array}{l}\text { Asadi and Zilouei } \\
(2017)\end{array}$ \\
\hline $\begin{array}{l}\text { Enterobacter cloacae } \\
\text { IIT-BT } 08\end{array}$ & Distillery effluent & Serum bottle & $37^{\circ} \mathrm{C}$ & $\begin{array}{l}\text { Initial = 7.5, } \\
\text { Operation = uncon- } \\
\text { trolled }\end{array}$ & $\begin{array}{l}7.38 \mathrm{~mol} \mathrm{H}_{2} / \mathrm{kg} \\
\mathrm{COD}_{\text {reduced }}\end{array}$ & Mishra and Das (2014) \\
\hline Bacillus cereus & Wheat straw & Batch & $37^{\circ} \mathrm{C}$ & $\begin{array}{l}\text { Initial = 7.5, } \\
\text { Operation = uncon- } \\
\text { trolled }\end{array}$ & $156.4 \mathrm{~mL} \mathrm{H} / \mathrm{g} V S$ & Saleem et al. (2020) \\
\hline $\begin{array}{l}\text { Ethanoligenens } \\
\text { harbinense B49 }\end{array}$ & Glucose & Serum bottle & $36^{\circ} \mathrm{C}$ & $\begin{array}{l}\text { Initial = 6.5, } \\
\text { Operation = uncon- } \\
\text { trolled }\end{array}$ & $113.5 \mathrm{mmol} \mathrm{H} / \mathrm{L}$ & Xu et al. (2016) \\
\hline $\begin{array}{l}\text { Ethanoligenens har- } \\
\text { binense YUAN-3 }\end{array}$ & Glucose & Batch & $35^{\circ} \mathrm{C}$ & $\begin{array}{l}\text { Initial }=7.0, \\
\text { Operation }=4.5\end{array}$ & $\begin{array}{l}2.62 \mathrm{~mol} \mathrm{H}_{2} / \mathrm{mol} \\
\text { glucose }\end{array}$ & Zhang et al. (2015) \\
\hline Escherichia coli & Glucose & Serum bottle & $37^{\circ} \mathrm{C}$ & ND & $\begin{array}{l}2.0 \mathrm{~mol} \mathrm{H}_{2} / \mathrm{mol} \\
\text { glucose }\end{array}$ & Bisaillon et al. (2006) \\
\hline $\begin{array}{l}\text { Janthinobacterium } \\
\text { agaricidamnosum }\end{array}$ & Glucose & Serological bottle & $25^{\circ} \mathrm{C}$ & $\begin{array}{l}\text { Initial = 6.5, } \\
\text { Operation = uncon- } \\
\text { trolled }\end{array}$ & $\begin{array}{l}0.86 \mathrm{~mol} \mathrm{H}_{2} / \mathrm{mol} \\
\text { glucose }\end{array}$ & $\begin{array}{l}\text { Alvarado-Cuevas et al. } \\
(2015)\end{array}$ \\
\hline $\begin{array}{l}\text { Polaromonas } \\
\text { jejuensis }\end{array}$ & Glucose & Serological bottle & $25^{\circ} \mathrm{C}$ & $\begin{array}{l}\text { Initial = 6.5, } \\
\text { Operation = uncon- } \\
\text { trolled }\end{array}$ & $\begin{array}{l}1.57 \mathrm{~mol} \mathrm{H}_{2} / \mathrm{mol} \\
\text { glucose }\end{array}$ & $\begin{array}{l}\text { Alvarado-Cuevas et al. } \\
(2015)\end{array}$ \\
\hline $\begin{array}{l}\text { Klebsiella pneumo- } \\
\text { niae }\end{array}$ & Brewery wastewater & $A \cap B B R$ & $35-36^{\circ} \mathrm{C}$ & $\begin{array}{l}\text { Initial }=5.5, \\
\text { Operation = uncon- } \\
\text { trolled }\end{array}$ & $\begin{array}{l}0.80-1.67 \mathrm{~mol} \mathrm{H}_{2} / \\
\text { mol glucose }\end{array}$ & Estevam et al. (2018) \\
\hline $\begin{array}{l}\text { Klebsiella pneumo- } \\
\text { niae BLb01 }\end{array}$ & Glycerol & Batch & $39^{\circ} \mathrm{C}$ & $\begin{array}{l}\text { Initial = 9.0, } \\
\text { Operation = uncon- } \\
\text { trolled }\end{array}$ & $45.0 \mathrm{~mol} \%$ & Costa et al. (2011) \\
\hline
\end{tabular}

ABR: anaerobic biodisc reactor; AnBBR: mechanically stirred anaerobic reactor; AnSBR: anaerobic sequenced-batch reactor; CSTR: continuous stirred tank reactor; TBSBR: trickling-bed sequenced-batch reactor; ND: no data; COD: chemical oxygen demand; TS: total solid; VS: volatile solid

cultures to reach the optimal production period can be time consuming (Kumar et al. 2018).

Mixed cultures have also been widely used (Table 2). Inoculum pre-treatment is necessary in a mixed culture system to enhance biohydrogen production yield by suppressing the activity of competing species, such as the biohydrogen-consumers and substrates competitor. Different pre-treatment methods will result in different starting microbial community structures. Zhang et al. (2011) investigated the effects of five different inoculum pre-treatment methods on mixed culture of aerobic seed sludge to enhance biohydrogen production from corn stover hydrolysate. Inoculum with no pre-treatment is composed of complex microbial community mainly represented by Enterobacter sp. and Pantoea sp. Pre-treatment using heat achieved the highest biohydrogen yield, 
Dzulkarnain et al. Bioresources and Bioprocessing (2022) 9:16

Page 9 of 25

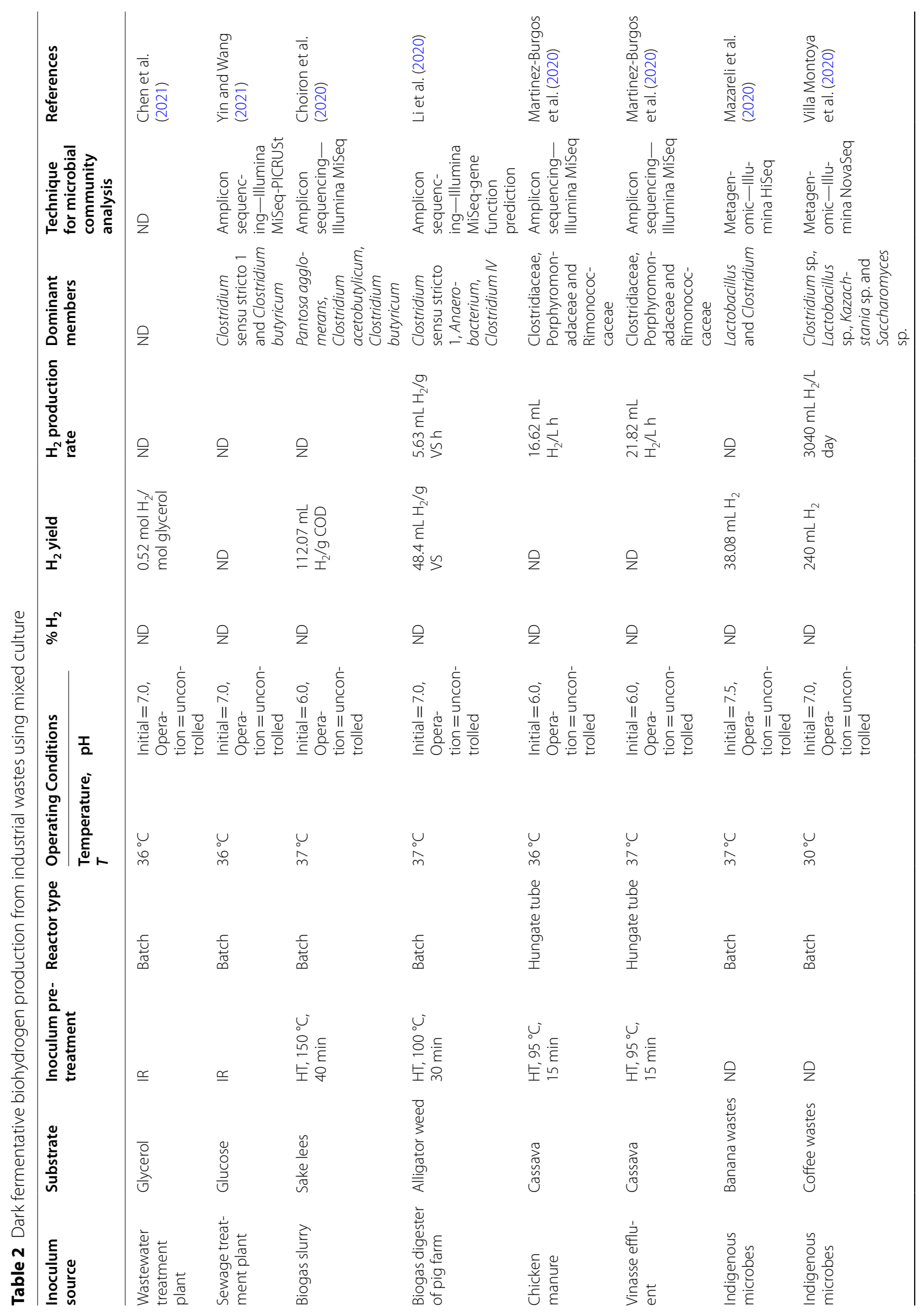




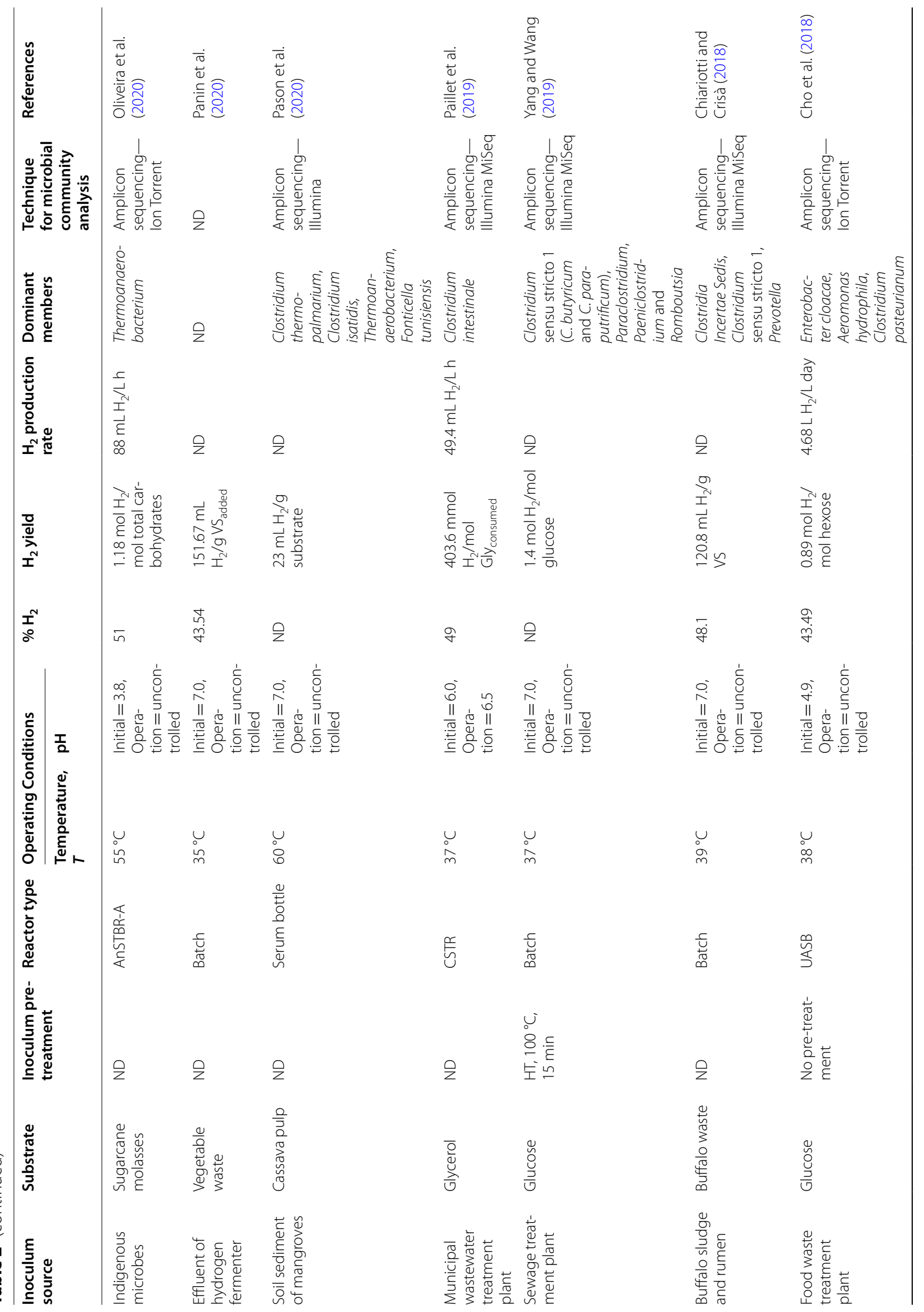




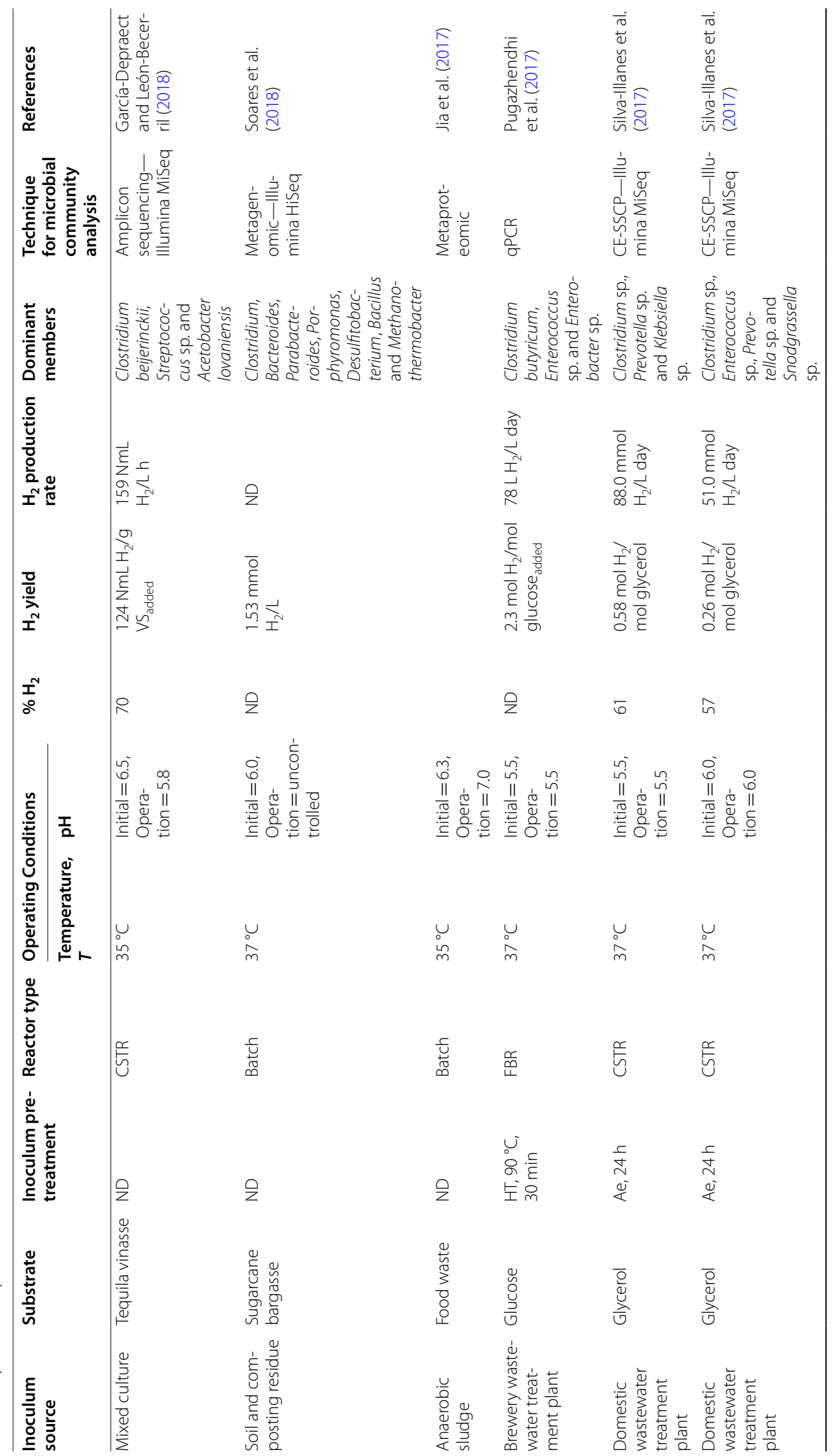




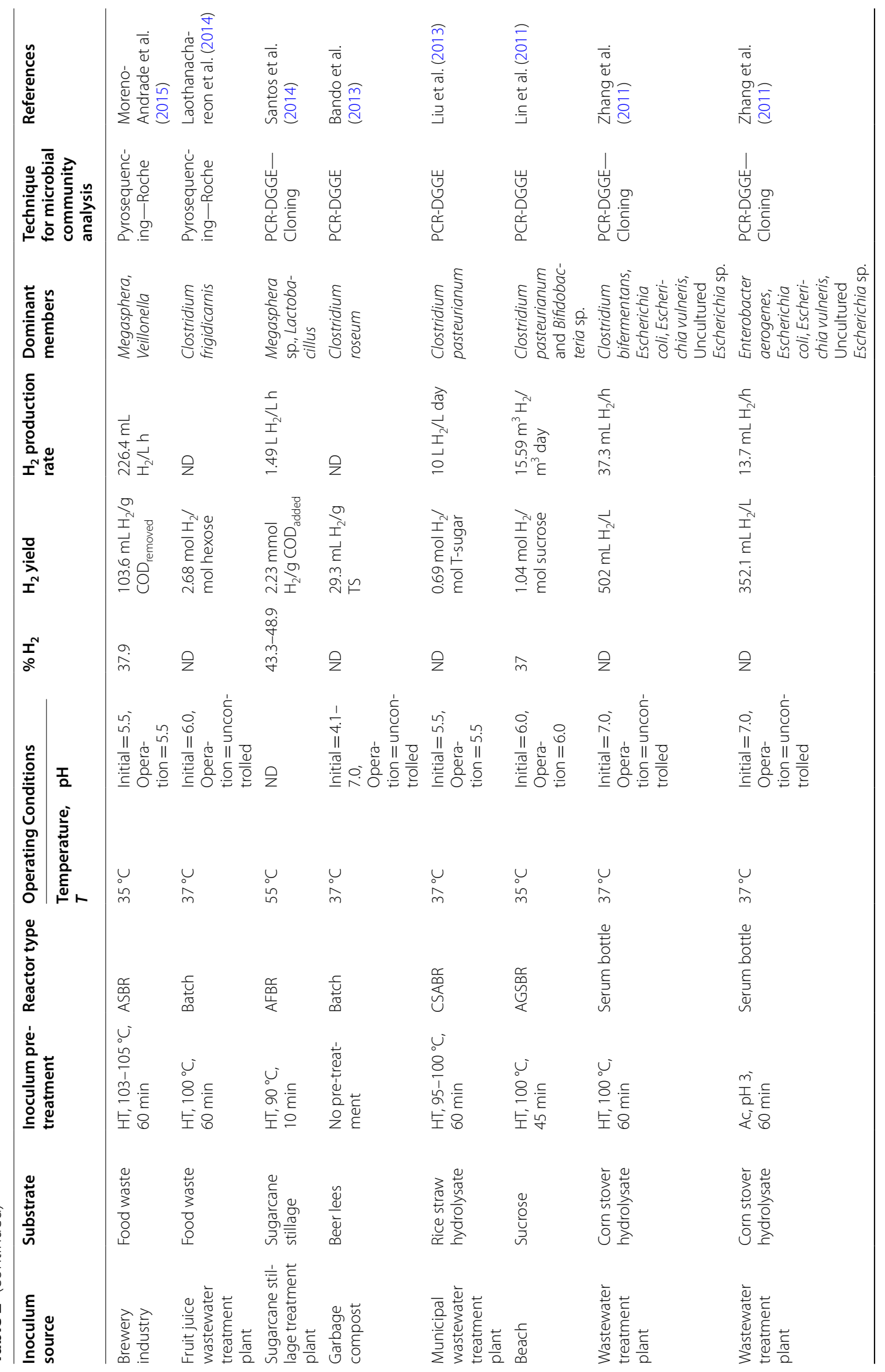




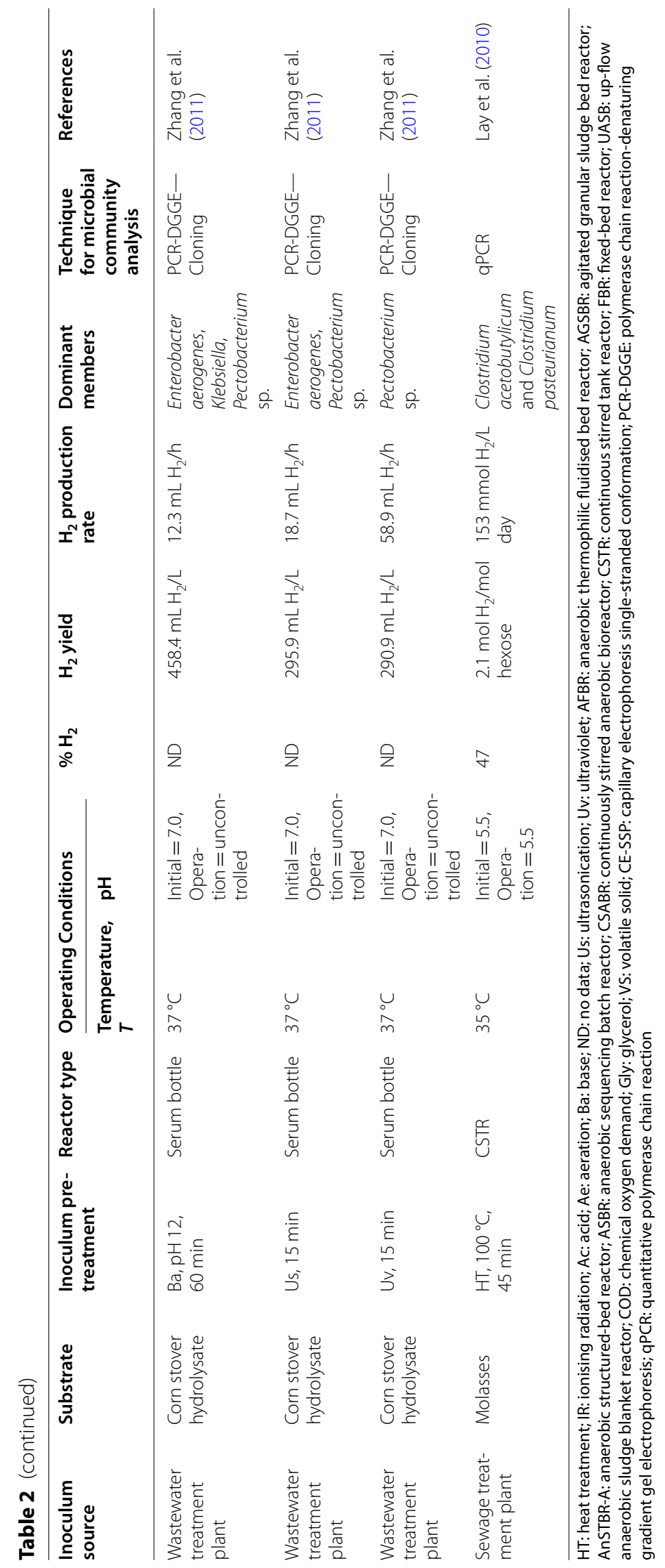


with the microbial community in the fermentation system dominated by $C$. bifermentans. Pre-treatment using base, acid, ultrasonic disruption and ultraviolet radiation favours facultative anaerobes, such as E. aerogenes, Klebsiella, Pectobacterium and E. coli. Heat pre-treatment is the commonly used inoculum pre-treatment method under mesophilic conditions (Table 2). It is usually selective to spore-forming species such as Clostridia, and inhibits other non-spore formers. In general, the use of mixed culture in dark fermentation has been shown to be promising, and it offer high hydrogen evolution rate and yields (Pachapur et al. 2019). However, understanding the metabolic complexities and process kinetics taking place within undefined microbiome systems are challenging.

Artificial microbial consortia containing selected microorganisms with specific metabolic or ecological functions has been shown to overcome the limitations of wild type and undefined microbiomes (Ergal et al. 2020). Recently, precision design of an artificial microbial consortia consisting E. aerogenes and C. acetobutylicum yielded $5.6 \mathrm{~mol} \mathrm{H}_{2} / \mathrm{mol}$ glucose. This was the highest biohydrogen yield reported so far, $40 \%$ beyond the Thauer limit (Ergal et al. 2020). The finding suggests that constructing a desired microbial consortium with well-studied biohydrogen-producing species will enable a comprehensive understanding of the microbial interactions, ease the control and balancing the effects of any perturbations. This will ultimately create a more efficient and robust engineered system.

\section{Palm oil mill effluent (POME) as substrate}

POME is the wastewater produced in large quantity during palm oil processing. It contains substantial amount of organic material, suspended solids, and oil and greases. Despite its nontoxic nature, POME is categorised as extremely high strength wastewater, which is 100 times more polluted than municipal sewage, and require effective treatment before discharge into the environment (Chia et al. 2020). Raw POME appears as thick brownish high colloidal suspension liquid mixture with a distinct offensive odour (Chia et al. 2020). It is characterised by high biological oxygen demand (BOD) (10, 250-80, $400 \mathrm{mg} / \mathrm{L})$, high chemical oxygen demand (COD) $(15,000-100,000 \mathrm{mg} / \mathrm{L})$, high oil and grease content $(130-18,000 \mathrm{mg} / \mathrm{L})$, high suspended solids (5000$54,000 \mathrm{mg} / \mathrm{L})$, high discharge temperature $\left(50-90^{\circ} \mathrm{C}\right)$ and is acidic (pH 3.4-6.9) (Audu et al. 2020). POME is rich with organic materials containing cellulose (11\%), hemicellulose (7\%) and lignin (42\%) (O-Thong et al. 2012). Given the high organic matter properties, recent POME treatment methods are coupled with bioenergy production and other value-added products, such as solvents, biomethane and biohydrogen.

Clostridia is the most commonly used genera for biohydrogen production from POME. C. butyricum has been used in several studies as pure culture inoculum for mesophilic batch biohydrogen production from POME via dark fermentation (Table 3). Singh et al. (2013b) observed that biohydrogen yield increased 1.5- to 2-fold when using an acclimatised immobilised C. butyricum. The immobilised cells recorded a biohydrogen yield of $5350 \mathrm{~mL} \mathrm{H}_{2} / \mathrm{L}$ POME with maximum biohydrogen production rate of $510 \mathrm{~mL} \mathrm{H}_{2} / \mathrm{L}$ POME/h. This species has also been reported to be the dominant biohydrogen producer in POME fermentation using mixed culture (Yossan et al. 2012) (Table 4). The effects of mesophilic and

Table 3 Dark fermentative biohydrogen production from POME using pure culture

\begin{tabular}{|c|c|c|c|c|c|}
\hline \multirow[t]{2}{*}{ Inoculum } & \multirow[t]{2}{*}{ Reactor type } & \multicolumn{2}{|c|}{ Operating conditions } & \multirow[t]{2}{*}{ Biohydrogen yield } & \multirow[t]{2}{*}{ References } \\
\hline & & Temperature, $T$ & $\mathrm{pH}$ & & \\
\hline Clostridium beijerinckii & Hungate tube & $30^{\circ} \mathrm{C}$ & $\begin{array}{l}\text { Initial }=7.0, \\
\text { Operation = uncontrolled }\end{array}$ & 4620 mL H ${ }_{2} / L$ medium & Rosa et al. (2020) \\
\hline Bacillus anthracis PUNAJAN 1 & CSTR & $35^{\circ} \mathrm{C}$ & $\begin{array}{l}\text { Initial =6.5, } \\
\text { Operation = uncontrolled }\end{array}$ & $236 \mathrm{ml} \mathrm{H}_{2} / \mathrm{g} \mathrm{COD}$ & Mishra et al. (2017) \\
\hline Escherichia coli & Serum bottle & $37^{\circ} \mathrm{C}$ & $\begin{array}{l}\text { Initial }=8.5, \\
\text { Operation = uncontrolled }\end{array}$ & $\begin{array}{l}0.66 \mathrm{~mol} \mathrm{H}_{2} / \mathrm{mol} \text { total monomeric } \\
\text { sugars }\end{array}$ & Taifor et al. (2017) \\
\hline Clostridium LS2 & UASB & $37^{\circ} \mathrm{C}$ & $\begin{array}{l}\text { Initial }=5.5 \\
\text { Operation }=5.5\end{array}$ & $380 \mathrm{~mL} \mathrm{H}_{2} / \mathrm{g} \mathrm{COD}$ & Singh et al. (2013b) \\
\hline Clostridium butyricum EB6 & Batch & $37^{\circ} \mathrm{C}$ & $\begin{array}{l}\text { Initial }=5.5 \\
\text { Operation }=5.5\end{array}$ & $5350 \mathrm{~mL} \mathrm{H} / \mathrm{L}$ POME & Singh et al. (2013c) \\
\hline Clostridium butyricum & Batch & $37^{\circ} \mathrm{C}$ & $\begin{array}{l}\text { Initial }=7.0, \\
\text { Operation }=5.5\end{array}$ & $2.18 \mathrm{~mol} \mathrm{H}_{2} / \mathrm{mol}$ total carbohydrate & Kamal et al. (2011) \\
\hline Clostridium butyricum EB6 & Batch & $37^{\circ} \mathrm{C}$ & $\begin{array}{l}\text { Initial }=5.5 \\
\text { Operation }=5.5\end{array}$ & $3195 \mathrm{~mL} \mathrm{H} / \mathrm{L}$ POME & Chong et al. (2009) \\
\hline
\end{tabular}




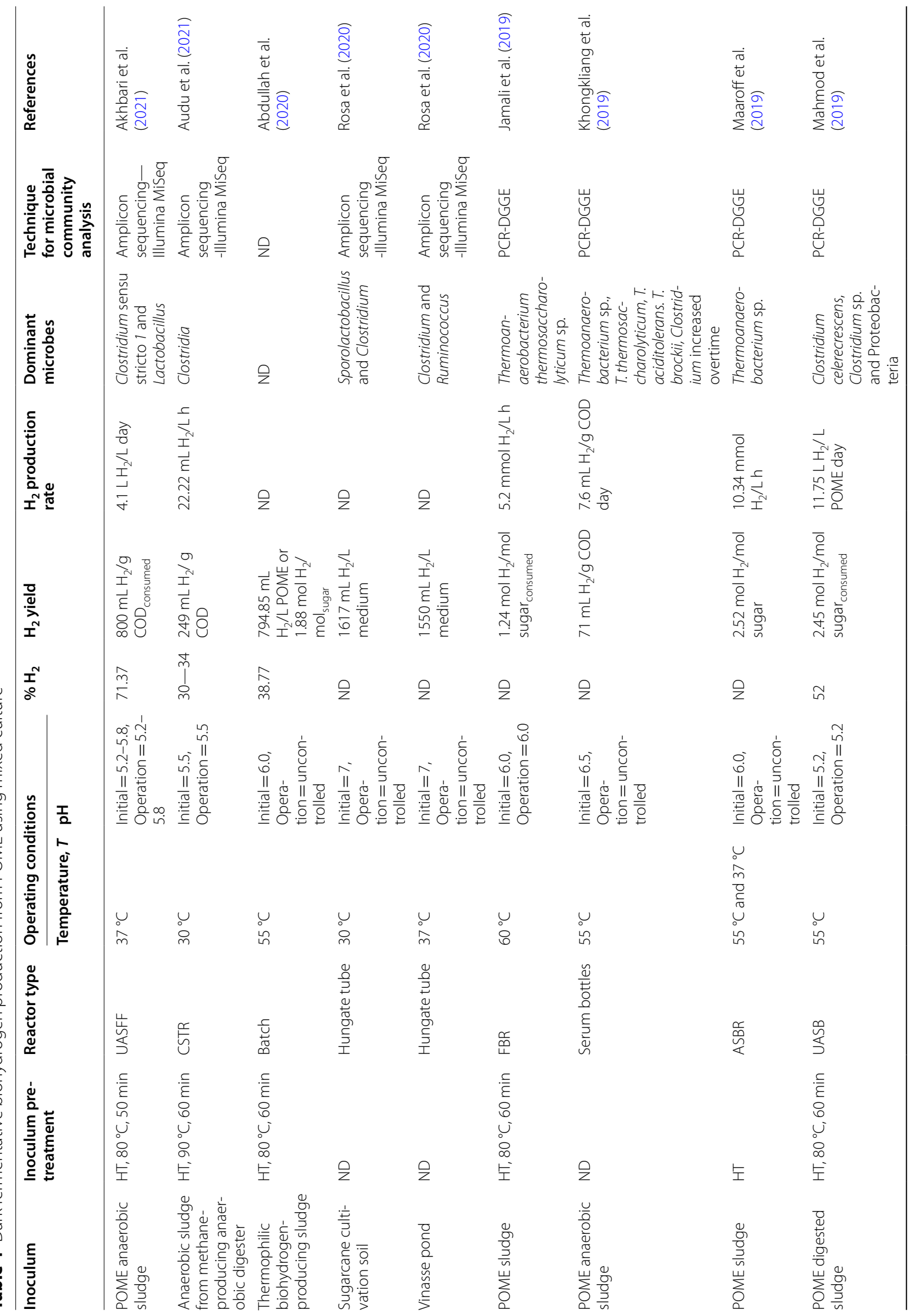




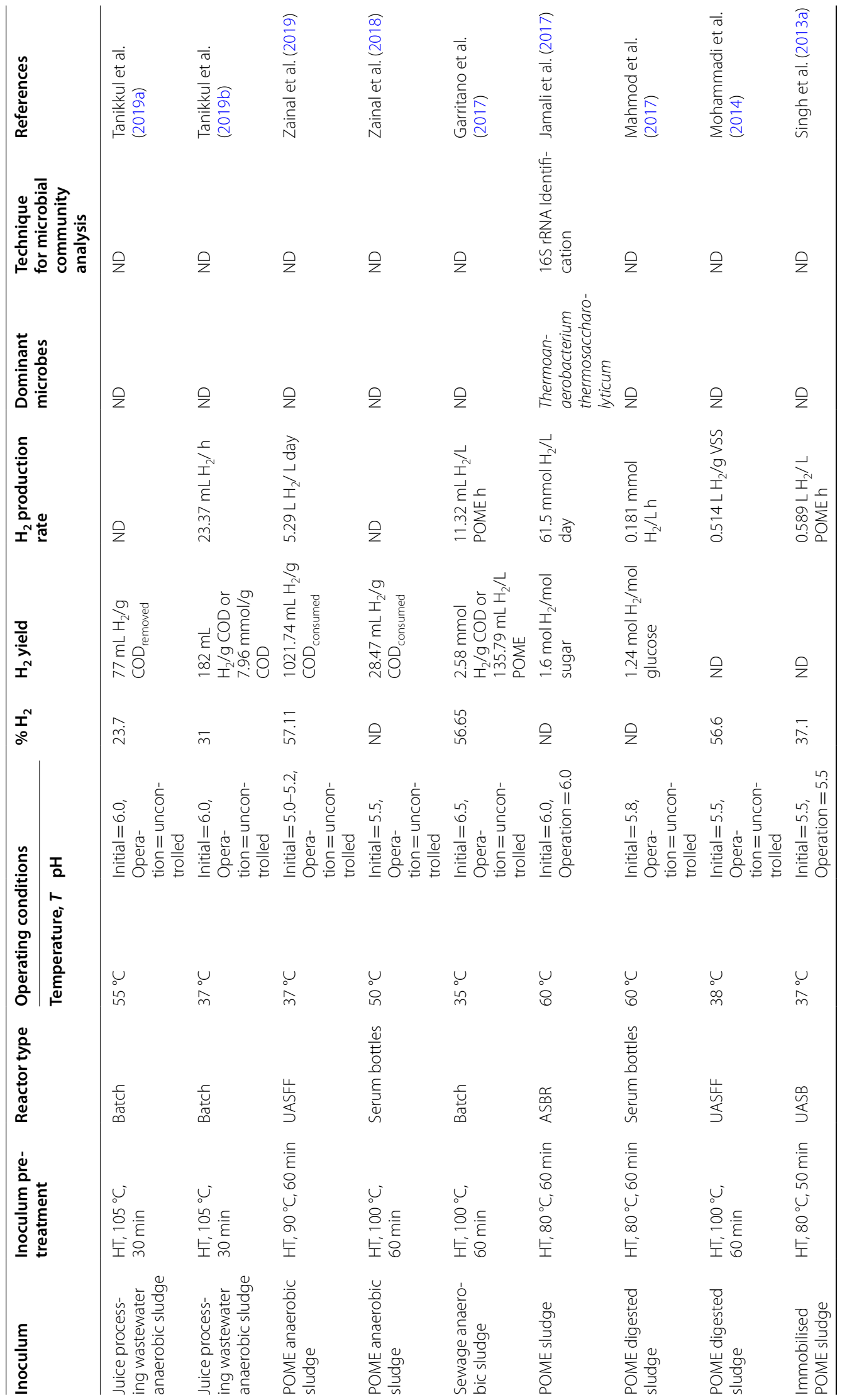




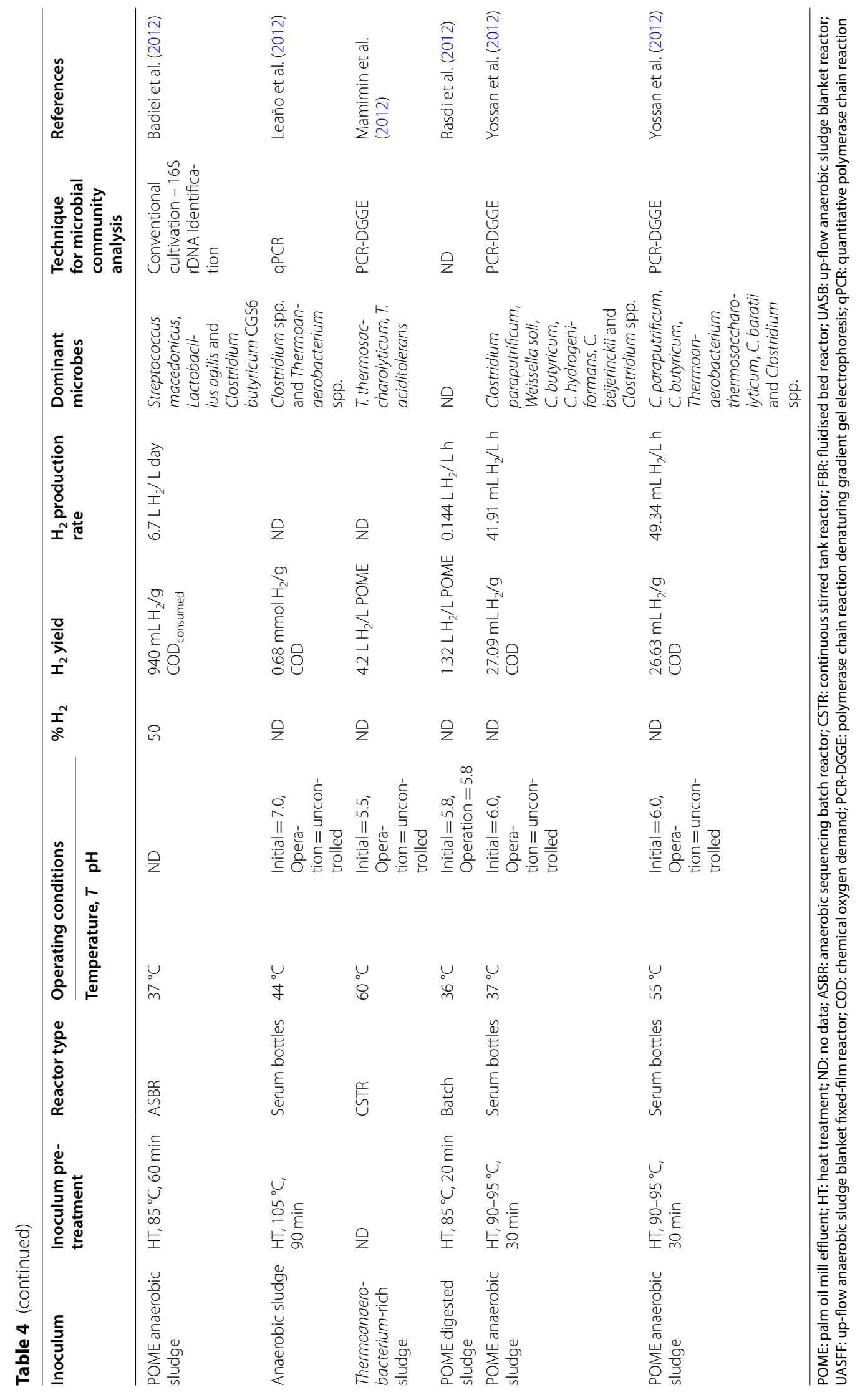


thermophilic conditions were also investigated, using anaerobic sludge as inoculum. Higher biohydrogen yield was achieved from mesophilic fermentation $(27.09 \mathrm{~mL} / \mathrm{g}$ COD) while higher biohydrogen production rate was achieved under thermophilic condition $\left(49.34 \mathrm{~mL} \mathrm{H}_{2} / \mathrm{L}\right.$ POME/h). Microbial community analysis performed showed that Clostridia dominated all the biohydrogen production systems operated at $25,37,45$ and $55^{\circ} \mathrm{C}$.

Different Clostridium species exhibit different metabolic activities, and their relative abundance vary depending on the operational conditions. Yossan et al. (2012) found that C. paraputrificum is the dominant member of the biohydrogen-producing community under all temperatures. In this study, C. butyricum was detected in the biohydrogen reactor operated at $37-55{ }^{\circ} \mathrm{C}$, whereas $\mathrm{C}$. beijerinckii and C. hydrogeniformans were only present at $37^{\circ} \mathrm{C}$. Biohydrogen production reactor at thermophilic condition was dominated by $C$. thermopalmarium, a non-cellulolytic biohydrogenproducing bacteria (Yossan et al. 2012). In another study, C. sensu stricto contributed $800 \mathrm{~mL} \mathrm{H}_{2} / \mathrm{g} \mathrm{COD}$ consumed of biohydrogen yield when treating POME with anaerobic sludge in up-flow anaerobic sludge blanket fixed-film (UASFF) reactor operated at $37{ }^{\circ} \mathrm{C}$ with the total abundance of $69.55 \%$ in the system (Akhbari et al. 2021). $C$. celerecrescens was the dominant biohydrogen producer in up-flow anaerobic sludge blanket (UASB) reactor using POME substrate operated under thermophilic condition (Mahmod et al. 2019). Clostridia can also be the main biohydrogen producers even though they do not dominate the whole community. Badiei et al. (2012) performed microbial community analysis on the anaerobic sludge of an anaerobic sequencing batch reactor (ASBR) operating under mesophilic temperature. $940 \mathrm{~mL} \mathrm{H}_{2} / \mathrm{g} \mathrm{COD}_{\text {removed }}$ of biohydrogen was obtained in this system. Only $20 \%$ of the relative microbial abundance were represented by Clostridia. The community was dominated by Streptococcus (50\% relative abundance) and Lactobacillus (30\% relative abundance), and the biohydrogen yield was comparable with the yield obtained by Akhbari et al. (2021) in a reactor dominated by Clostridium. This suggests that Clostridia does not have to dominate the system in order to obtain a high biohydrogen production yield. Deep metagenomics sequencing can help reveal the syntrophic relationship that may exist between Clostridia and the other genera not known to be the biohydrogen producers, and the connections between the different communities at different trophic levels in the reactor.

\section{Tools for biohydrogen microbiome analysis}

Biohydrogen production through dark fermentation from organic wastes, including POME, is a complex biochemical process, carried out by microbial communities with a range of relationships between them. Dark fermentation can be divided into four key stages which are hydrolysis, acidogenesis, acetogenesis and methanogenesis (section "Microbiomes in dark fermentative biohydrogen production"). Methanogenesis is often suppressed and undesired in biohydrogen production. These processes occur synergistically in a successive manner and each stage is facilitated by a distinct guild of microorganisms. A robust and efficient dark fermentation system requires a delicate balance of microbial population dynamics and metabolic activities among different guilds or trophic groups of the biohydrogen-producing microbiomes. Understanding of the microbial ecology of the dark fermentation process can help to improve the performance towards maximising biohydrogen production, and ensure that this process is economically feasible.

A range of techniques have been used in characterising the complex biohydrogen-producing microbial communities, from conventional cultivation-dependent approaches to cultivation-independent approaches. The advanced multi-omics technologies are also increasingly being use for this purpose. Cultivation-dependent method have contributed in the discovery of many key microbial species in biohydrogen-producing bioreactors from organic industrial waste and POME (AlvaradoCuevas et al. 2015; Harun et al. 2012; Hsieh et al. 2016; Mishra et al. 2017; Noparat et al. 2012; Singh et al. 2014; Yin and Wang 2017; Zhang et al. 2015). While economical and a generally useful method to shed light on some key members, not many can be characterised this way, particularly when a system-based approach is required. Some key taxa also have their syntrophic partners belonging to different functional guilds (Lim et al. 2020). This method is further limited by species-specific morphological variations since some microorganisms share similar morphological, physiological or biochemical characteristics which makes the classification challenging (Lim et al. 2020). While cultivation method might be time consuming and labour intensive, it is the only technique to characterise a specific strain in detail. Currentomics technologies also require more reference genomes to evaluate the biohydrogen-producing microbiomes sequence data. Therefore, culture-dependent method will remain essential for studying the microbial diversity in biohydrogen-producing microbiomes. Recently, novel biohydrogen-producing bacteria, Clostridium sartagoforme NASGE 01 and Enterobacter cloacae NASGE 02 were isolated from sago industrial effluent using this method (Nizzy et al. 2020).

Advancement in molecular biology and DNA sequencing techniques has enabled various culture-independent methods to be used to study the microbiomes in biohydrogen-producing reactors. Denaturing gradient gel 
electrophoresis (DGGE) and single-strand conformation polymorphism (SSCP) are among the microbiome fingerprinting techniques used to evaluate and compare different microbiomes in dark fermentation from organic wastes and POME. Both techniques involve polymerase chain reaction (PCR) amplification of a hypervariable region of the 16S rRNA gene and migration of the PCR product fragments on polyacrylamide gel that will provide different banding patterns, which reflect the structure of microbial communities and species abundance. Using PCR-DGGE, the genus Megasphaera sp. was identified as the main biohydrogen producer with $14 \%$ relative abundance, in thermophilic dark fermentation reactor of sugarcane stillage inoculated with granular sludge of a sugarcane stillage treatment plant. Clostridia were not detected in this system (Santos et al. 2014). While in dark fermentative biohydrogen production of beer lees inoculated with non-pre-treated garbage compost, using PCR-DGGE, C. roseum was found to be the prevalent biohydrogen producers in all high biohydrogen-producing batch fermentations, whereas C. perfringens and $C$. sporogenes were detected in low biohydrogen-producing batch fermentations (Bando et al. 2013). The presence of Bifidobacterium spp. and Lactobacillus spp. inhibited biohydrogen production through substrate competition with biohydrogen producers. Biohydrogen-producing species such as $C$. butyricum and $C$. tyrobutyricum were also found as the substrate competitors in biohydrogen fermenter dominated by C. pasteurianum (Lin et al. 2011). When POME was used as substrate, PCR-DGGE is still among the commonly used methods in studying the biohydrogen-producing microbiomes. The genus Thermoanaerobacterium, such as T. thermosaccharolyticum, was often reported as the main biohydrogen producers in thermophilic POME dark fermentation using POME anaerobic sludge as inoculum source (Jamali et al. 2019; Khongkliang et al. 2019; Maaroff et al. 2019).

The use of SSCP to investigate biohydrogen-producing microbial community structure is still limited. Using SSCP, operational $\mathrm{pH}$ of a continuous stirred tank reactor (CSTR) fed with glycerol was found to change the structure of the dominant microbial populations (Silva-Illanes et al. 2017). Hydraulic retention time (HRT) changed the metabolic pattern and the composition of subdominant microorganisms such as Enterococcus, Prevotella, Sutterella, Pseudomonas and Acinetobacter, ultimately affecting the ability of the consortium to produce biohydrogen. In general, DGGE and SSCP are not quantitative, more labour intensive, time consuming, prone to PCR biases and has low resolution in complex microbiome profiles (Kumar et al. 2018). Nevertheless, these microbiome fingerprinting techniques could remain useful for quick screening purposes, and to acquire a glimpse of biohydrogen-producing microbiomes from a large number of samples.

Quantitative PCR (qPCR) has also been used in studying several biohydrogen reactors using organic wastes (including POME) as substrates, to quantify the changes of specific microbial populations (Lay et al. 2010; Leaño et al. 2012; Pugazhendhi et al. 2017). In contrast to the common PCR which is qualitative, qPCR can accurately quantify the copy number of genes of interest in a sample by measuring the fluorescence of a specific probe used for amplification (Lim et al. 2020; Tolvanen and Karp 2011). This technique eliminates post-PCR target analysis, cheaper and offers a fast, accurate and simple approach for high-throughput analysis (Nurmi et al. 2002). Individual taxa or guilds in biohydrogen microbiomes can also be quantified using fluorescent in situ hybridisation (FISH) technique. In FISH, cells of interest is hybridised with a specific fluorogenic oligonucleotide probes and its relative abundance is then measured by quantifying the ratio of the hybridised cells to the total cell count using a fluorescence microscope. FISH probes Tbm1282, Ccs432 and Tbmthsac184 specific for detection of Thermoanaerobacterium, Caldicellulosiruptor and T. thermosaccharolyticum have been designed and used to assess the microbial composition in thermophilic and extreme thermophilic biohydrogen-producing reactors fed with POME, lignocellulosic hydrolysate and synthetic sugars (O-Thong et al. 2008). FISH overcomes the limitations of PCR-based molecular techniques. Nevertheless, cell hybridisation is time consuming, making FISH less suitable for high-throughput community structure investigation (Ravenschlag et al. 2001). Detection of novel microorganisms may also be challenging, the probe design and selection require some information on the community structure prior to the analysis (Lim et al. 2020).

High throughput next generation -omics technologies are increasingly being employed to better understand the complex microbiomes driving dark fermentative biohydrogen production. Amplicon sequencing, metagenomics and metaproteomics have all been employed in this context. Amplicon sequencing also known as metaprofiling is a culture-independent technique to profile the taxonomic diversity, structure and composition of a microbiome based on a marker gene (Escobar-Zepeda et al. 2015). 16S rRNA genes have been exclusively used as a marker gene for library preparation through PCR amplification in studies of microbial communities, including biogas-producing microbiomes (Sharpton 2014; Tonge et al. 2014). Using amplicon sequencing method, biohydrogen production using sake lees was found to be enhanced when the microbial community in the system changed from Bacillus muralis and B. cereus as the 
dominant taxa, to Pantoea agglomerans, C. acetobutylicum and C. butyricum (Choiron et al. 2020). Besides, with amplicon sequencing, Sporolactobacillus was the dominant taxa with relative abundance $97 \%$ in the fermentation of POME using microbial consortia from sugarcane cultivation soil (Rosa et al. 2020). Sporolactobacillus is an anaerobic facultative bacterium producing lactic acid. Although its role in biohydrogen production is unknown, its metabolic by-products could be used as substrates for biohydrogen production by other microorganisms. Amplicon sequencing is commonly done on an Illumina MiSeq platform (Akhbari et al. 2021; Audu et al. 2021; Martinez-Burgos et al. 2020; Yang and Wang 2019) while Ion Torrent platform has also been used in several studies (Cho et al. 2018; Oliveira et al. 2020). A few studies have also attempted to predict the community functions from amplicon sequencing of biohydrogen microbiomes using bioinformatic tools, such as PICRUSt (Li et al. 2020; Yin and Wang 2021) mostly using other organic wastes. So far, this has not been reported for POME. Amplicon sequencing is the best and economical option to understand the microbial community members in general, but it has limitations and may result in biases (Lim et al. 2020), leading to the increasing applications of shotgun metagenomics.

Shotgun metagenomics independently sequences total genomic DNA retrieved directly from a sample to produce reads that align to various genomic locations for the countless genomes present, including the non-microbes (Sharpton 2014). Metagenomic tools could unravel the vast taxonomic diversity, metabolic function potential and physiology of uncultivated microorganisms, including the novel and rare taxa, and previously unknown metabolic pathways (Vanwonterghem et al. 2014). A few studies have investigated the biohydrogen-producing microbiomes using metagenomics (Mazareli et al. 2020; Soares et al. 2018; Villa Montoya et al. 2020). Mazareli et al. (2020) used metagenomics to correlate taxonomic diversity of indigenous microbial biomass with the performance of biohydrogen reactor fed with banana wastes under mesophilic temperature. Using FMAP (Functional Mapping and Analysis Pipeline) for metagenomic and metatranscriptomic studies tool, Clostridium and Lactobacillus were the dominant indigenous acidogenic bacteria, and the main genes encoding key enzymes involved in the fermentation were found to be related to carbohydrate metabolism, acidogenesis and biohydrogen production enzymes such as glucose-6-phosphate dehydrogenase, fructokinase, lactate dehydrogenase and pyruvate ferredoxin oxidoreductase. Metagenomic study by Villa Montoya et al. (2020) reported domain Bacteria represented $97.2 \%$ relative abundance with the predominance of genera Clostridium (87.9\% relative abundance) in the mesophilic biohydrogen-producing bioreactor fed with coffee wastes. Gene identifications showed that $8.3 \%$ of the genes were corresponded to anaerobic degradation enzymes mainly for the production of organic acids and alcohols and may be associated with the metabolic potential of Clostridium sp. In addition, 37 KEGG orthologues (KOs) were identified to be associated with biohydrogen production, highlighting enzymes pyruvate-ferredoxin oxidoreductase, anaerobic carbon-monoxide dehydrogenase, formate dehydrogenase and ferredoxin hydrogenase. Genes related to these enzymes were mainly found in Clostridium sp. (Villa Montoya et al. 2020).

Breakthroughs in next generation sequencing (NGS) technologies has also led to another subfield of -omic technologies, which is metaproteomics. Metaproteomics profiles enzymes and proteins in microbiomes, and can potentially link the function of a protein to a taxon and its metabolic activities (Chistoserdova 2009; Lim et al. 2020). Metaproteomics has been widely applied in studying anaerobic digester bioreactors and human gut microbiome, but its application is still limited in investigating the microbiomes of biohydrogen dark fermentation. Previously, metaproteomics was used to establish the relationship between phylogeny, function, and metabolic activity of biohydrogen and methane co-production microbiomes from food waste (Jia et al. 2017). A total of 651 bacterial proteins and 477 archaeal proteins were detected in the study, revealing the complexity and metabolic diversity during the biogas production process. The study also revealed that the key bacterial proteins from Gammaproteobacteria, Clostridia and Bacilli related to biohydrogen production came from pyruvic acid decarboxylase and formic acid decomposition pathway in carbohydrate metabolisms.

\section{Future outlook}

Biogas (i.e. methane) microbiomes are more widely and intensively studied than biohydrogen, despite the fact that these two processes share many biochemical and metabolic routes. This is probably due the more advanced research and wider adoption of anaerobic digestion for biogas production as cleaner energy production technology in the society. It has been demonstrated that methane production is directly linked to the composition of the anaerobic digester microbiomes, in addition to the microbial metabolism, which is dependent on the environmental parameters of the reactor (Campanaro et al. 2020). This makes understanding of the microbial composition of a bioreactor and their behaviour a critical aspect in the quest for a feasible biohydrogen production via dark fermentation. Pugazhendhi et al. (2019) reviewed the microbiomes involved in the anaerobic hydrogen-producing 
granules (HPG). Granulation increases the reaction efficiency of a fermenter, compared to using sludge. The dominant taxa in the microbial community of reactor systems employing HPG has been discussed, allowing the monitoring of the microbial species for easier control of the kinetic parameters, and contributes to the development of stable bioprocess system (Pugazhendhi et al. 2019). This suggests the importance of meta-analysis of hydrogen-producing microbial community from different reactor systems, and the correlation with their physicochemical parameters and reactor performance.

A summary of the molecular tools in analysing the biohydrogen-producing microbial community has recently been published (Kumar et al. 2020), describing the "targeted" molecular tools (e.g. FISH, qPCR) and the advantages of NGS in providing quicker and more comprehensive investigation. A combination of culture-dependent approach, targeted molecular tools and NGS, and multi-omics are definitely the way forward in providing a system-based understanding of the biohydrogen microbiomes. Multi-omics of this engineered reactor system can also benefit from the rapidly expanding experimental and computational tools for investigating human and environmental microbiomes, allowing for deeper understanding of the community structure and functions from the -omics data. This includes the advancements in co-occurrence network, genome-scale metabolic model, protein-protein interaction network, the metabolic-driven metabolomics network (Liu et al. 2020), and the integration of all the -omics data. This is in addition to the need for best practices for analysing the microbiomes towards a unified approach in the analysis of reactor systems.

Knowledge obtained from the -omics techniques can be used to engineer a desired community structure, towards maximising productivity of an engineered system, and balancing the effects of any perturbations. Tools for manipulating community structure in situ are also being investigated. CRISPR/Cas-related system has been used in a targeted genome editing of specific microorganisms within a complex microbial community (Rubin et al. 2020), paving the way for manipulation of microbiomes in many different applications, possibly in the biogas and biohydrogen-producing reactor system too. There is still a long way to go before this precise gene and genome manipulation system can be applied in a complex community like the anaerobic digester's, but it is important to first have the full understanding of the microbial community and the relationships with the physicochemical parameters in controlling the production yield and rate.

\section{Conclusion}

Biohydrogen is a common by-product of many bacterial metabolic pathways during dark fermentation. Microbial communities involved in dark fermentation are phylogenetically and functionally diverse which contribute to biohydrogen production from the breakdown of complex organic substrates, such as POME and other industrial wastes. As a system which relies on microbial metabolisms, insights on the microbial members present in the reactor is important towards obtaining a robust and efficient biohydrogen production system. Numerous molecular tools for screening, quantification and identification of biohydrogen-producing microbial communities have been used to correlate the phylogeny, interspecies interactions and their function to dark fermentative biohydrogen process. Currently, DGGE and amplicon sequencing are widely used in the study of biohydrogen microbiomes. The use of -omics technologies in biohydrogen research are still relatively limited, compared to the more widely investigated anaerobic digester's microbiomes for biomethane production. We believe similar advanced tools can be applied to biohydrogen-producing reactors too, with the prospect to unravel the limitless potential of the microbial members in the system.

\section{Abbreviations}

POME: Palm oil mill effluent; rRNA: Ribosomal RNA; RDP: Ribosomal Database Project; NCBI: National Center for Biotechnology Information; MAGs: Metagenome-assembled genomes; MiDAS: Microbial Database for Activated Sludge; ASV: Amplicon sequence variant; $\mathrm{CO}_{2}$ : Carbon dioxide; $\mathrm{H}_{2}$ : Hydrogen; $\mathrm{CH}_{4}$ : Methane; SCFA: Short-chain fatty acid; PFL: Pyruvate formate lyase; Fd: Ferredoxin; PFOR: Pyruvate ferredoxin oxidoreductase; NADH: Nicotinamide adenine dinucleotide; NFOR: Nicotinamide adenine dinucleotide ferredoxin oxidoreductase; ATP: Adenosine triphosphate; BOD: Biological oxygen demand; COD: Chemical oxygen demand; UASFF: Up-flow anaerobic sludge blanket fixed-film; UASB: Up-flow anaerobic sludge blanket; ASBR: Anaerobic sequencing batch reactor; DGGE: Denaturing gradient gel electrophoresis; SSCP: Single-strand conformation polymorphism; PCR: Polymerase chain reaction; CSTR: Continuous stirred tank reactor; HRT: Hydraulic retention time; QPCR: Quantitative polymerase chain reaction; FISH: Fluorescent in situ hybridisation; FMAP: Functional Mapping and Analysis Pipeline; KOs: KEGG orthologues; NGS: Next generation sequencing; HPG: Hydrogen-producing granules.

\section{Acknowledgements}

Not applicable.

\section{Authors' contributions}

ELND and MFA-W designed the content. ELND and JOA performed literature search and analysis. JOA designed the figures in the manuscript. ELND, JOA and MFA-W wrote the manuscript. MFA-W and WRZWD provided expertise and revised the manuscript. All authors read and approved the final manuscript.

\section{Funding}

ELND would like to acknowledge The Ministry of Higher Education (MOHE) Malaysia for providing financial support through MyBrainSc scholarship.JOA would like to acknowledge the Nigerian Tertiary Education Trust Fund for the scholarship provided. MFA-W would like to acknowledge the funding received from UTM via Transdisciplinary Research Grant no. 05G24 and UTMShine Grant No. 09G86. 
Availability of data and materials

Not applicable.

\section{Declarations}

\section{Ethics approval and consent to participate}

Not applicable.

\section{Consent for publication}

Not applicable.

\section{Competing interests}

The authors declare that no competing interests involved.

\section{Author details}

${ }^{1}$ Department of Biosciences, Faculty of Science, Universiti Teknologi Malaysia, 81310 Skudai, Johor, Malaysia. ${ }^{2}$ Department of Science Laboratory Technology, Modibbo Adama University, PMB 2076, Yola, Adamawa, Nigeria.

${ }^{3}$ Taiwan-Malaysia Innovation Centre for Clean Water and Sustainable Energy

(WISE Centre), Universiti Teknologi Malaysia, 81310 Skudai, Johor, Malaysia.

\section{Received: 6 December 2021 Accepted: 14 February 2022}

\section{Published online: 05 March 2022}

\section{References}

Abdullah MF et al (2020) Effect of carbon/nitrogen ratio and ferric ion on the production of biohydrogen from palm oil mill effluent (POME). Biocatal Agric Biotechnol 23:101445. https://doi.org/10.1016/j.bcab.2019.101445

Abendroth C et al (2015) Eubacteria and archaea communities in seven mesophile anaerobic digester plants in Germany. Biotechnol Biofuels 8:1-10. https://doi.org/10.1186/s13068-015-0271-6

Akhbari A et al (2021) Start-up study of biohydrogen production from palm oil mill effluent in a lab-scale up-flow anaerobic sludge blanket fixed-film reactor. Int J Hydrogen Energy. https://doi.org/10.1016/j.jijydene.2020. 12.125

Alvarado-Cuevas ZD et al (2015) Biohydrogen production using psychrophilic bacteria isolated from Antarctica. Int J Hydrogen Energy 40:7586-7592. https://doi.org/10.1016/j.jijhydene.2014.10.063

Alvarez-Guzmán CL et al (2020) Biohydrogen production from cheese whey powder by Enterobacter asburiae: effect of operating conditions on hydrogen yield and chemometric study of the fermentative metabolites. Energy Rep 6:1170-1180. https://doi.org/10.1016/j.egyr.2020.04. 038

Asadi N, Zilouei H (2017) Optimization of organosolv pretreatment of rice straw for enhanced biohydrogen production using Enterobacter aerogenes. Biores Technol 227:335-344. https://doi.org/10.1016/j.biort ech.2016.12.073

Audu JO et al (2020) Dark fermentation and bioelectrochemical systems for enhanced biohydrogen production from palm oil mill effluent: current progress, potentials, and future perspectives. In: Zakaria ZA, Boopathy $R$ Dib JR (eds) Valorisation of agro-industrial residues_Volume I: Biological approaches. Springer, Cham, pp 1-35

Audu JO et al (2021) Optimization of the operational parameters for mesophilic biohydrogen production from palm oil mill effluent using enriched mixed culture. Biomass Convers Biorefinery. https://doi.org/ 10.1007/s13399-021-01488-9

Azman NF et al (2016) Biohydrogen production from de-oiled rice bran as sustainable feedstock in fermentative process. Int J Hydrogen Energy 41:145-156. https://doi.org/10.1016/j.ijhydene.2015.10.018

Badiei M et al (2012) Microbial community analysis of mixed anaerobic microflora in suspended sludge of ASBR producing hydrogen from palm oil mill effluent. Int J Hydrogen Energy 37:3169-3176. https://doi.org/10. 1016/j.ijhydene.2011.11.063

Bando $Y$ et al (2013) A microbiological study of biohydrogen production from beer lees. Int J Hydrogen Energy 38:2709-2718. https://doi.org/10. 1016/j.jihydene.2012.11.142

Beckers L et al (2013) Improving effect of metal and oxide nanoparticles encapsulated in porous silica on fermentative biohydrogen production by Clostridium butyricum. Biores Technol 133:109-117. https://doi.org/10.1016/j.biortech.2012.12.168

Berg G et al (2020) Microbiome definition re-visited: old concepts and new challenges. Microbiome 8:1-22. https://doi.org/10.1186/ s40168-020-00875-0

Bisaillon A et al (2006) The effect of nutrient limitation on hydrogen production by batch cultures of Escherichia coli. Int J Hydrogen Energy 31:1504-1508. https://doi.org/10.1016/j.ijhydene.2006.06.016

Cabrol L et al (2017) Microbial ecology of fermentative hydrogen producing bioprocesses: useful insights for driving the ecosystem function. FEMS Microbiol Rev 41:158-181. https://doi.org/10.1093/femsre/ fuw043

Campanaro S et al (2016) Metagenomic analysis and functional characterization of the biogas microbiome using high throughput shotgun sequencing and a novel binning strategy. Biotechnol Biofuels 9:1-17. https://doi.org/10.1186/s13068-016-0441-1

Campanaro S et al (2020) New insights from the biogas microbiome by comprehensive genome-resolved metagenomics of nearly 1600 species originating from multiple anaerobic digesters. Biotechnol Biofuels 13:25. https://doi.org/10.1186/s13068-020-01679-y

Castellano-Hinojosa A et al (2018) New concepts in anaerobic digestion processes: recent advances and biological aspects. Appl Microbiol Biotechnol 102:5065-5076. https://doi.org/10.1007/ s00253-018-9039-9

Chang $S$ et al (2011) Evaluation of different pretreatment methods for preparing hydrogen-producing seed inocula from waste activated sludge. Renew Energy 36:1517-1522. https://doi.org/10.1016/j.renene.2010. 11.023

Chen $Y$ et al (2021) Comparison of fermentative hydrogen production from glycerol using immobilized and suspended mixed cultures. Int J Hydrogen Energy. https://doi.org/10.1016/j.ijhydene.2021.01.003

Chia WY et al (2020) Outlook on biorefinery potential of palm oil mill effluent for resource recovery. J Environ Chem Eng 8:104519. https://doi.org/10. 1016/j.jece.2020.104519

Chiariotti A, Crisà A (2018) Bio-hydrogen production from buffalo waste with rumen inoculum and metagenomic characterization of bacterial and archaeal community. Front Sustain Food Syst. https://doi.org/10.3389/ fsufs.2018.00013

Chistoserdova $L$ (2009) Functional metagenomics: recent advances and future challenges. Biotechnol Genet Eng Rev 26:335-352. https://doi.org/10. 5661/bger-26-335

Cho S-K et al (2018) Effects of low-strength ultrasonication on dark fermentative hydrogen production: start-up performance and microbial community analysis. Appl Energy 219:34-41. https://doi.org/10.1016/j. apenergy.2018.03.047

Choiron M et al (2020) Biohydrogen production improvement using hot compressed water pretreatment on sake brewery waste. Int J Hydrogen Energy 45:17220-17232. https://doi.org/10.1016/j.ijhydene.2020.04.199

Chong M-L et al (2009) Biohydrogen production by Clostridium butyricum EB6 from palm oil mill effluent. Int J Hydrogen Energy 34:764-771. https:// doi.org/10.1016/j.ijhydene.2008.10.095

Conklin A et al (2006) Growth kinetics and competition between Methanosarcina and Methanosaeta in mesophilic anaerobic digestion. Water Environ Res 78:486-496. https://doi.org/10.2175/106143006×95393

Costa JB et al (2011) The optimization of biohydrogen production by bacteria using residual glycerol from biodiesel synthesis. J Environ Sci Health Part A 46:1461-1468. https://doi.org/10.1080/10934529.2011.609036

Dada O et al (2013) Biohydrogen production from ricebran using Clostridium saccharoperbutylacetonicum N1-4. Int J Hydrogen Energy 38:1506315073. https://doi.org/10.1016/j.jihydene.2013.07.048

Das D (2017) A road map on biohydrogen production from organic wastes. INAE Letters 2:153-160. https://doi.org/10.1007/s41403-017-0031-y

Dueholm MS et al (2021) MiDAS 4: a global catalogue of full-length 16S rRNA gene sequences and taxonomy for studies of bacterial communities in wastewater treatment plants. bioRxiv. https://doi.org/10.1101/2021.07. 06.451231

Ergal i et al (2020) Biohydrogen production beyond the Thauer limit by precision design of artificial microbial consortia. Commun Biol 3:443. https:// doi.org/10.1038/s42003-020-01159-x 
Escobar-Zepeda A et al (2015) The road to metagenomics: from microbiology to DNA sequencing technologies and bioinformatics. Front Genet 6:348. https://doi.org/10.3389/fgene.2015.00348

Estevam A et al (2018) Production of biohydrogen from brewery wastewater using Klebsiella pneumoniae isolated from the environment. Int J Hydrogen Energy 43:4276-4283. https://doi.org/10.1016/j.ijhydene. 2018.01 .052

García-Depraect O, León-Becerril E (2018) Fermentative biohydrogen production from tequila vinasse via the lactate-acetate pathway: operational performance, kinetic analysis and microbial ecology. Fuel 234:151-160. https://doi.org/10.1016/j.fuel.2018.06.126

Garritano AdN et al (2017) Efficient biohydrogen production via dark fermentation from hydrolyzed palm oil mill effluent by non-commercial enzyme preparation. Int J Hydrogen Energy 42:29166-29174. https:// doi.org/10.1016/j.ijhydene.2017.10.025

Ghimire A et al (2015) A review on dark fermentative biohydrogen production from organic biomass: process parameters and use of by-products. Appl Energy 144:73-95. https://doi.org/10.1016/j.apene rgy.2015.01.045

Gonzalez-Martinez A et al (2016) Archaeal and bacterial community dynamics and bioprocess performance of a bench-scale two-stage anaerobic digester. Appl Microbiol Biotechnol 100:6013-6033. https://doi. org/10.1007/s00253-016-7393-z

Harun I et al (2012) Hydrogen production performance by Enterobacter cloacae $\mathrm{KBH} 3$ isolated from termite guts. Int J Hydrogen Energy 37:15052-15061. https://doi.org/10.1016/j.ijhydene.2012.07.101

Hassa J et al (2018) Metagenome, metatranscriptome, and metaproteome approaches unraveled compositions and functional relationships of microbial communities residing in biogas plants. Appl Microbiol Biotechnol 102:5045-5063. https://doi.org/10.1007/s00253-018-8976-7

Hay JXW et al (2013) Biohydrogen production through photo fermentation or dark fermentation using waste as a substrate: overview, economics, and future prospects of hydrogen usage. Biofuels Bioprod Biorefin 7:334-352. https://doi.org/10.1002/bbb.1403

Hsieh P-H et al (2016) Explore the possible effect of $\mathrm{TiO} 2$ and magnetic hematite nanoparticle addition on biohydrogen production by Clostridium pasteurianum based on gene expression measurements. Int J Hydrogen Energy 41:21685-21691. https://doi.org/10.1016/j. ijhydene.2016.06.197

Jamali NS et al (2017) Particle size variations of activated carbon on biofilm formation in thermophilic biohydrogen production from palm oil mill effluent. Energy Convers Manage 141:354-366. https://doi.org/10. 1016/j.enconman.2016.09.067

Jamali NS et al (2019) Thermophilic biohydrogen production from palm oil mill effluent: effect of immobilized cells on granular activated carbon in fluidized bed reactor. Food Bioprod Process 117:231-240. https://doi. org/10.1016/j.fbp.2019.07.012

Jia X et al (2017) Metaproteomic analysis of the relationship between microbial community phylogeny, function and metabolic activity during biohydrogen-methane coproduction under short-term hydrothermal pretreatment from food waste. Biores Technol 245:1030-1039. https:// doi.org/10.1016/j.biortech.2017.08.180

Kamal S et al (2011) Pre-treatment effect of palm oil mill effluent (POME) during hydrogen production by a local isolate Clostridium butyricum. Int J Adv Sci Eng Inf Technol 2:54-60. https://doi.org/10.18517/ijaseit.2.4.214

Kanchanasuta S et al (2017) Stability of Clostridium butyricum in biohydrogen production from non-sterile food waste. Int J Hydrogen Energy 42:3454-3465. https://doi.org/10.1016/j.ijhydene.2016.09.111

Kaza S et al (2018) What a waste 2.0: a global snapshot of solid waste management to 2050. World Bank Publications, Washington

Keskin T et al (2019) Effect of percolation frequency on biohydrogen production from fruit and vegetable wastes by dry fermentation. Int $J$ Hydrogen Energy 44:18767-18775. https://doi.org/10.1016/j.j.jhydene. 2018.12.099

Khongkliang P et al (2019) High efficient biohydrogen production from palm oil mill effluent by two-stage dark fermentation and microbial electrolysis under thermophilic condition. Int J Hydrogen Energy 44:3184131852. https://doi.org/10.1016/j.ijhydene.2019.10.022

Kivistö A et al (2013) Non-sterile process for biohydrogen and 1,3-propanediol production from raw glycerol. Int J Hydrogen Energy 38:11749-11755. https://doi.org/10.1016/j.jhydene.2013.06.119
Kraemer JT, Bagley DM (2007) Improving the yield from fermentative hydrogen production. Biotech Lett 29:685-695. https://doi.org/10.1007/ s10529-006-9299-9

Kumar $\mathrm{G}$ et al (2018) Insights into evolutionary trends in molecular biology tools in microbial screening for biohydrogen production through dark fermentation. Int J Hydrogen Energy 43:19885-19901. https://doi.org/ 10.1016/j.ijhydene.2018.09.040

Kumar G et al (2020) Application of molecular techniques in biohydrogen production as a clean fuel. Sci Total Environ 722:137795. https://doi.org/ 10.1016/j.scitotenv.2020.137795

Laothanachareon T et al (2014) Analysis of microbial community adaptation in mesophilic hydrogen fermentation from food waste by tagged $16 \mathrm{~S}$ rRNA gene pyrosequencing. J Environ Manage 144:143-151. https:// doi.org/10.1016/j.jenvman.2014.05.019

Laurent B et al (2012) Effects of hydrogen partial pressure on fermentative biohydrogen production by a chemotropic Clostridium bacterium in a new horizontal rotating cylinder reactor. Energy Procedia 29:34-41. https://doi.org/10.1016/j.egypro.2012.09.006

Lay C-H et al (2010) Biohydrogen production from soluble condensed molasses fermentation using anaerobic fermentation. Int J Hydrogen Energy 35:13445-13451. https://doi.org/10.1016/j.ijhydene.2009.11.128

Leaño EP et al (2012) Ultrasonic pretreatment of palm oil mill effluent: impact on biohydrogen production, bioelectricity generation, and underlying microbial communities. Int J Hydrogen Energy 37:12241-12249. https://doi.org/10.1016/j.ijhydene.2012.06.007

Li W et al (2017) Two-phase anaerobic digestion of municipal solid wastes enhanced by hydrothermal pretreatment: viability, performance and microbial community evaluation. Appl Energy 189:613-622. https://doi. org/10.1016/j.apenergy.2016.12.101

Li Z et al (2018) Anaerobic co-digestion of sewage sludge and food waste for hydrogen and VFA production with microbial community analysis. Waste Manage 78:789-799. https://doi.org/10.1016/j.wasman.2018.06. 046

$\mathrm{Li} \mathrm{H}$ et al (2020) Effects of harvest month on biochemical composition of alligator weed for biohydrogen and biomethane cogeneration: identifying critical variations in microbial communities. Int J Hydrogen Energy 45:4161-4173. https://doi.org/10.1016/j.ijhydene.2019.11.208

Lim JW et al (2020) Chapter one - the microbiome driving anaerobic digestion and microbial analysis. In: LiY, Khanal SK (eds) Advances in bioenergy. Elsevier, Amserdam, pp 1-61

Lin C-Y et al (2011) A pilot-scale high-rate biohydrogen production system with mixed microflora. Int J Hydrogen Energy 36:8758-8764. https:// doi.org/10.1016/j.ijhydene.2010.07.115

Liu C-M et al (2013) Biohydrogen production evaluation from rice straw hydrolysate by concentrated acid pre-treatment in both batch and continuous systems. Int J Hydrogen Energy 38:15823-15829. https:// doi.org/10.1016/j.ijhydene.2013.07.055

Liu H et al (2018) Characteristics of hydrogen-producing enrichment cultures from marine sediment using macroalgae Laminaria japonica as a feedstock. J Biosci Bioeng 126:710-714. https://doi.org/10.1016/j.jbiosc. 2018.05.012

Liu Z et al (2020) Network analyses in microbiome based on high-throughput multi-omics data. Brief Bioinform 22:1639-1655. https://doi.org/10. 1093/bib/bbaa005

Łukajtis R et al (2018) Hydrogen production from biomass using dark fermentation. Renew Sustain Energy Rev 91:665-694. https://doi.org/10.1016/j. rser.2018.04.043

Maaroff RM et al (2019) Biohydrogen production from palm oil mill effluent (POME) by two stage anaerobic sequencing batch reactor (ASBR) system for better utilization of carbon sources in POME. Int J Hydrogen Energy 44:3395-3406. https://doi.org/10.1016/j.jijydene.2018.06.013

Mahato RK et al (2020) Biohydrogen production from fruit waste by Clostridium strain BOH3. Renew Energy 153:1368-1377. https://doi.org/ 10.1016/.renene.2020.02.092

Mahmod SS et al (2017) Pretreatment conditions of palm oil mill effluent (POME) for thermophilic biohydrogen production by mixed culture. Int J Hydrogen Energy 42:27512-27522. https://doi.org/10.1016/j.ijhydene. 2017.07.178

Mahmod SS et al (2019) Operation performance of up-flow anaerobic sludge blanket (UASB) bioreactor for biohydrogen production by self-granulated sludge using pre-treated palm oil mill effluent (POME) as carbon 
source. Renew Energy 134:1262-1272. https://doi.org/10.1016/j.renene. 2018.09.062

Mamimin C et al (2012) Simultaneous thermophilic hydrogen production and phenol removal from palm oil mill effluent by Thermoanaerobacteriumrich sludge. Int J Hydrogen Energy 37:15598-15606. https://doi.org/10. 1016/j.ijhydene.2012.04.062

Martinez-Burgos WJ et al (2020) Biohydrogen production in cassava processing wastewater using microbial consortia: process optimization and kinetic analysis of the microbial community. Biores Technol 309:123331. https://doi.org/10.1016/j.biortech.2020.123331

Mazareli RC et al (2020) Metagenomic analysis of autochthonous microbial biomass from banana waste: Screening design of factors that affect hydrogen production. Biomass Bioenergy 138:105573. https://doi.org/ 10.1016/j.biombioe.2020.105573

Mcllroy SJ et al (2015) MiDAS: the field guide to the microbes of activated sludge. Database. https://doi.org/10.1093/database/bav062

Menzel T et al (2020) Role of microbial hydrolysis in anaerobic digestion. Energies 13:5555. https://doi.org/10.3390/en13215555

Mishra P, Das D (2014) Biohydrogen production from Enterobacter cloacae IIT-BT 08 using distillery effluent. Int J Hydrogen Energy 39:7496-7507. https://doi.org/10.1016/j.ijhydene.2013.08.100

Mishra P et al (2017) Fermentative hydrogen production from indigenous mesophilic strain Bacillus anthracis PUNAJAN 1 newly isolated from palm oil mill effluent. Int J Hydrogen Energy 42:16054-16063. https:// doi.org/10.1016/j.jijhydene.2017.05.120

Mishra P et al (2019) Outlook of fermentative hydrogen production techniques: an overview of dark, photo and integrated dark-photo fermentative approach to biomass. Energ Strat Rev 24:27-37. https://doi.org/ 10.1016/j.esr.2019.01.001

Mohammadi P et al (2014) High-rate fermentative hydrogen production from palm oil mill effluent in an up-flow anaerobic sludge blanket-fixed film reactor. Chem Eng Res Des 92:1811-1817. https://doi.org/10.1016/j. cherd.2014.04.023

Mohammed A et al (2018) Biohydrogen production by antarctic psychrotolerant Klebsiella sp. ABZ11. Pol J Microbiol 67:283. https://doi.org/10. 21307/pjm-2018-033

Moreno-Andrade I et al (2015) Biohydrogen from food waste in a discontinuous process: Effect of HRT and microbial community analysis. Int J Hydrogen Energy 40:17246-17252. https://doi.org/10.1016/j.ijhydene. 2015.04.084

Nitipan S et al (2014) Microbial community analysis of thermophilic mixed culture sludge for biohydrogen production from palm oil mill effluent. Int J Hydrogen Energy 39:19285-19293. https://doi.org/10.1016/j.ijhyd ene.2014.05.139

Nizzy AM et al (2020) Identification of hydrogen gas producing anaerobic bacteria isolated from sago industrial effluent. Curr Microbiol 77:25442553. https://doi.org/10.1007/s00284-020-02092-2

Noparat $P$ et al (2012) Potential for using enriched cultures and thermotolerant bacterial isolates for production of biohydrogen from oil palm sap and microbial community analysis. Int J Hydrogen Energy 37:1641216420. https://doi.org/10.1016/j.ijhydene.2012.02.103

Nurmi J et al (2002) High-performance real-time quantitative RT-PCR using lanthanide probes and a dual-temperature hybridization assay. Anal Chem 74:3525-3532. https://doi.org/10.1021/ac020093y

Oliveira CA et al (2020) Thermophilic biohydrogen production from sugarcane molasses under low pH: metabolic and microbial aspects. Int J Hydrogen Energy 45:4182-4192. https://doi.org/10.1016/j.ijhydene.2019.12. 013

Ortigueira J et al (2015) Third generation biohydrogen production by Clostridium butyricum and adapted mixed cultures from Scenedesmus obliquus microalga biomass. Fuel 153:128-134. https://doi.org/10. 1016/j.fuel.2015.02.093

O-Thong S (2017) Microbial population optimization for control and improvement of dark hydrogen fermentation. In: Jozala AF (ed) Fermentation processes. IntechOpen, London

O-Thong S, et al (2008) $16 \mathrm{~S}$ rRNA-targeted probes for specific detection of Thermoanaerobacterium spp., Thermoanaerobacterium thermosaccharolyticum, and Caldicellulosiruptor spp. by fluorescent in situ hybridization in biohydrogen producing systems. Int J Hydrogen Energy 33:60826091. https://doi.org/10.1016/j.ijhydene.2008.07.094
O-Thong S, et al (2012) Thermophilic anaerobic co-digestion of oil palm empty fruit bunches with palm oil mill effluent for efficient biogas production. Appl Energy 93:648-654. https://doi.org/10.1016/j.apenergy.2011.12. 092

Pachapur VL et al (2019) Seed pretreatment for increased hydrogen production using mixed-culture systems with advantages over pure-culture systems. Energies 12:530. https://doi.org/10.3390/en12030530

Paillet F et al (2019) Improvement of biohydrogen production from glycerol in micro-oxidative environment. Int J Hydrogen Energy 44:17802-17812. https://doi.org/10.1016/j.ijhydene.2019.05.082

Panin S et al (2020) Biohydrogen and biogas production from mashed and powdered vegetable residues by an enriched microflora in dark fermentation. Int J Hydrogen Energy. https://doi.org/10.1016/j.ijhydene. 2020.09.246

Pason P et al (2020) One-step biohydrogen production from cassava pulp using novel enrichment of anaerobic thermophilic bacteria community. Biocatal Agric Biotechnol 27:101658. https://doi.org/10.1016/j. bcab.2020.101658

Patel SKS et al (2018) Beyond the theoretical yields of dark-fermentative biohydrogen. Indian J Microbiol 58:529-530. https://doi.org/10.1007/ s12088-018-0759-4

Plangklang P et al (2012) Enhanced bio-hydrogen production from sugarcane juice by immobilized Clostridium butyricum on sugarcane bagasse. Int J Hydrogen Energy 37:15525-15532. https://doi.org/10.1016/j.ijhydene. 2012.02.186

Pugazhendhi A et al (2017) Process performance of biohydrogen production using glucose at various HRTs and assessment of microbial dynamics variation via q-PCR. Int J Hydrogen Energy 42:27550-27557. https://doi. org/10.1016/j.jphydene.2017.06.184

Pugazhendhi A et al (2019) Microbiome involved in anaerobic hydrogen producing granules: a mini review. Biotechnol Reports 21:e00301-e00301. https://doi.org/10.1016/j.btre.2018.e00301

Puhulwella RG et al (2014) Mesophilic biohydrogen production by Clostridium butyricum CWBI1009 in trickling biofilter reactor. Int J Hydrogen Energy 39:16902-16913. https://doi.org/10.1016/i.ijhydene.2014.08.087

Rambabu K et al (2020) Enhanced biohydrogen production from date seeds by Clostridium thermocellum ATCC 27405. Int J Hydrogen Energy 45:22271-22280. https://doi.org/10.1016/j.ijhydene.2019.06.133

Rambabu K et al (2021) Augmented biohydrogen production from rice mill wastewater through nano-metal oxides assisted dark fermentation. Biores Technol 319:124243. https://doi.org/10.1016/j.biortech.2020. 124243

Rasdi Z et al (2012) Kinetic analysis of biohydrogen production from anaerobically treated POME in bioreactor under optimized condition. Int J Hydrogen Energy 37:17724-17730. https://doi.org/10.1016/j.ijhydene. 2012.08.095

Ravenschlag K et al (2001) Quantitative molecular analysis of the microbial community in marine Arctic sediments (Svalbard). Appl Environ Microbiol 67:387-395. https://doi.org/10.1128/AEM.67.1.387-395.2001

Rosa D et al (2020) Biological hydrogen production from palm oil mill effluent (POME) by anaerobic consortia and Clostridium beijerinckii. J Biotechnol 323:17-23. https://doi.org/10.1016/j.jbiotec.2020.06.015

Rubin BE et al (2020) Targeted genome editing of bacteria within microbial communities. bioRxiv. https://doi.org/10.1101/2020.07.17.209189

Saleem A et al (2020) Fermentation of simple and complex substrates to biohydrogen using pure Bacillus cereus RTUA and RTUB strains. Environ Technol Innov 18:100704. https://doi.org/10.1016/j.eti.2020.100704

Santos SC et al (2014) Organic loading rate impact on biohydrogen production and microbial communities at anaerobic fluidized thermophilic bed reactors treating sugarcane stillage. Biores Technol 159:55-63. https://doi.org/10.1016/j.biortech.2014.02.051

Saravanan A et al (2021) Biohydrogen from organic wastes as a clean and environment-friendly energy source: production pathways, feedstock types, and future prospects. Biores Technol 342:126021. https://doi.org/ 10.1016/j.biortech.2021.126021

Sarma S et al (2019) Homologous overexpression of hydrogenase and glycerol dehydrogenase in Clostridium pasteurianum to enhance hydrogen production from crude glycerol. Biores Technol 284:168-177. https:// doi.org/10.1016/j.biortech.2019.03.074

Sen B, Suttar RR (2012) Mesophilic fermentative hydrogen production from sago starch-processing wastewater using enriched mixed cultures. Int J 
Hydrogen Energy 37:15588-15597. https://doi.org/10.1016/j.jjydene. 2012.04.027

Seon $J$ et al (2014) Bacterial community structure in maximum volatile fatty acids production from alginate in acidogenesis. Biores Technol 157:22-27. https://doi.org/10.1016/j.biortech.2014.01.072

Sharma S et al (2020) Waste-to-energy nexus for circular economy and environmental protection: recent trends in hydrogen energy. Sci Total Environ 713:136633. https://doi.org/10.1016/j.scitotenv.2020.136633

Sharpton TJ (2014) An introduction to the analysis of shotgun metagenomic data. Front Plant Sci 5:209. https://doi.org/10.3389/fpls.2014.00209

Sieber JR et al (2012) Genomic insights into syntrophy: the paradigm for anaerobic metabolic cooperation. Annu Rev Microbiol 66:429-452. https://doi. org/10.1146/annurev-micro-090110-102844

Sikora A et al (2017) Anaerobic digestion: I. A common process ensuring energy flow and the circulation of matter in ecosystems. II. A tool for the production of gaseous biofuels. Ferment Processes 14:271. https://doi.org/10. $5772 / 64645$

Silva-Illanes F et al (2017) Impact of hydraulic retention time (HRT) and pH on dark fermentative hydrogen production from glycerol. Energy 141:358367. https://doi.org/10.1016/j.energy.2017.09.073

Singh L et al (2013a) Biohydrogen production from palm oil mill effluent using immobilized mixed culture. J Ind Eng Chem 19:659-664. https://doi.org/ 10.1016/j.jiec.2012.10.001

Singh L et al (2013b) Application of immobilized upflow anaerobic sludge blanket reactor using Clostridium LS2 for enhanced biohydrogen production and treatment efficiency of palm oil mill effluent. Int J Hydrogen Energy 38:2221-2229. https://doi.org/10.1016/i.jihydene.2012.12.004

Singh L et al (2013c) Biohydrogen production from palm oil mill effluent using immobilized Clostridium butyricum EB6 in polyethylene glycol. Process Biochem 48:294-298. https://doi.org/10.1016/j.procbio.2012.12.007

Singh S et al (2014) Biohydrogen production by Thermoanaerobacterium thermosaccharolyticum TERI S7 from oil reservoir flow pipeline. Int J Hydrogen Energy 39:4206-4214. https://doi.org/10.1016/i.i.jhydene.2013.12.179

Soares LA et al (2018) Metagenomic analysis and optimization of hydrogen production from sugarcane bagasse. Biomass Bioenerg 117:78-85. https:// doi.org/10.1016/j.biombioe.2018.07.018

Song W et al (2020) Improving biohydrogen production through dark fermentation of steam-heated acid pretreated Alternanthera philoxeroides by mutant Enterobacter aerogenes ZJU1. Sci Total Environ 716:134695. https:// doi.org/10.1016/j.scitotenv.2019.134695

Stolze Y et al (2016) Identification and genome reconstruction of abundant distinct taxa in microbiomes from one thermophilic and three mesophilic production-scale biogas plants. Biotechnol Biofuels 9:1-18. https://doi. org/10.1186/s13068-016-0565-3

Taifor AF et al (2017) Elucidating substrate utilization in biohydrogen production from palm oil mill effluent by Escherichia coli. Int J Hydrogen Energy 42:5812-5819. https://doi.org/10.1016/j.ijhydene.2016.11.188

Tanikkul P et al (2019a) Thermophilic biohydrogen recovery from palm oil mill effluent. Int J Hydrogen Energy 44:5176-5181. https://doi.org/10.1016/j. ijhydene.2018.10.005

Tanikkul P et al (2019b) Ozonation aided mesophilic biohydrogen production from palm oil mill effluent. Int J Hydrogen Energy 44:5182-5188. https:// doi.org/10.1016/j.ijhydene.2018.09.180

Tian Q-Q et al (2015) Enhanced biohydrogen production from sugarcane bagasse by Clostridium thermocellum supplemented with $\mathrm{CaCO}$. Biores Technol 197:422-428. https://doi.org/10.1016/j.biortech.2015.08.111

Tolvanen KES, Karp MT (2011) Molecular methods for characterizing mixed microbial communities in hydrogen-fermenting systems. Int J Hydrogen Energy 36:5280-5288. https://doi.org/10.1016/j.ijhydene.2011.01.029

Tonge DP et al (2014) Amplicon-based metagenomic analysis of mixed fungal samples using proton release amplicon sequencing. PLoS ONE 9:e93849. https://doi.org/10.1371/journal.pone.0093849

Ulhiza TA et al (2018) Optimization of culture conditions for biohydrogen production from sago wastewater by Enterobacter aerogenes using Response Surface Methodology. Int J Hydrogen Energy 43:22148-22158. https://doi. org/10.1016/ji.jhydene.2018.10.057

Vanwonterghem I et al (2014) Linking microbial community structure, interactions and function in anaerobic digesters using new molecular techniques. Curr Opin Biotechnol 27:55-64. https://doi.org/10.1016/j.copbio. 2013.11 .004
Vardar-Schara G et al (2008) Metabolically engineered bacteria for producing hydrogen via fermentation. Microb Biotechnol 1:107-125. https://doi.org/ 10.1111/j.1751-7915.2007.00009.x

Venkiteshwaran K et al (2015) Relating anaerobic digestion microbial community and process function: supplementary issue: water microbiology. Microbiol Insights 8:37-44. https://doi.org/10.4137/MBI.S33593

Viana MB et al (2019) The source of inoculum and the method of methanogenesis inhibition can affect biological hydrogen production from crude glycerol. BioEnergy Res 12:733-742. https://doi.org/10.1007/ s12155-019-09994-5

Villa Montoya AC et al (2020) Optimization of key factors affecting hydrogen production from coffee waste using factorial design and metagenomic analysis of the microbial community. Int J Hydrogen Energy 45:4205-4222. https://doi.org/10.1016/j.ijhydene.2019.12.062

Wang J, Yin Y (2019) Progress in microbiology for fermentative hydrogen production from organic wastes. Crit Rev Environ Sci Technol 49:825-865. https:/ doi.org/10.1080/10643389.2018.1487226

Wang Y-Y et al (2011) Effects of various pretreatment methods of anaerobic mixed microflora on biohydrogen production and the fermentation pathway of glucose. Int J Hydrogen Energy 36:390-396. https://doi.org/ 10.1016/j.jhydene.2010.09.092

Wang P et al (2018) Microbial characteristics in anaerobic digestion process of food waste for methane production — a review. Biores Technol 248:29-36. https://doi.org/10.1016/j.biortech.2017.06.152

Whang L-M et al (2011) Metabolic and energetic aspects of biohydrogen production of Clostridium tyrobutyricum: the effects of hydraulic retention time and peptone addition. Biores Technol 102:8378-8383. https://doi.org/10. 1016/j.biortech.2011.03.101

Whipps J et al (1988) Mycoparasitism and plant disease control. In: Burge M (ed) Fungi in biological control systems. Manchester University Press, Manchester, pp 161-187

Xu J-F et al (2016) Buffering action of acetate on hydrogen production by Ethanoligenens harbinense B49. Electron J Biotechnol 23:7-11. https://doi.org/ 10.1016/j.ejbt.2016.07.002

Yang G, Wang J (2019) Changes in microbial community structure during dark fermentative hydrogen production. Int J Hydrogen Energy 44:2554225550. https://doi.org/10.1016/j.ijhydene.2019.08.039

Yin Y, Wang J (2017) Isolation and characterization of a novel strain Clostridium butyricum INET1 for fermentative hydrogen production. Int J Hydrogen Energy 42:12173-12180. https://doi.org/10.1016/j.jihydene.2017.02.083

Yin Y, Wang J (2021) Predictive functional profiling of microbial communities in fermentative hydrogen production system using PICRUSt. Int J Hydrogen Energy 46:3716-3725. https://doi.org/10.1016/j.ijhydene.2020.10.246

Yossan S et al (2012) Effect of initial pH, nutrients and temperature on hydrogen production from palm oil mill effluent using thermotolerant consortia and corresponding microbial communities. Int J Hydrogen Energy 37:13806-13814. https://doi.org/10.1016/j.jijydene.2012.03.151

Zainal BS et al (2018) Effects of process, operational and environmental variables on biohydrogen production using palm oil mill effluent (POME). Int J Hydrogen Energy 43:10637-10644. https://doi.org/10.1016/j.jhydene. 2017.10.167

Zainal BS et al (2019) UASFF start-up for biohydrogen and biomethane production from treatment of palm oil mill effluent. Int J Hydrogen Energy 44:20725-20737. https://doi.org/10.1016/j.jijhydene.2018.07.037

Zhang K et al (2011) Effects of various pretreatment methods on mixed microflora to enhance biohydrogen production from corn stover hydrolysate. J Environ Sci 23:1929-1936. https://doi.org/10.1016/S1001-0742(10) 60679-1

Zhang L et al (2015) Effects of the ecological factors on hydrogen production and [Fe-Fe]-hydrogenase activity in Ethanoligenens harbinense YUAN-3. Int J Hydrogen Energy 40:6792-6797. https://doi.org/10.1016/j.jhydene. 2015.02.015

Zhang Y et al (2020) Effect of enzymolysis time on biohydrogen production from photo-fermentation by using various energy grasses as substrates. Biores Technol 305:123062. https://doi.org/10.1016/j.biortech.2020.123062

\section{Publisher's Note}

Springer Nature remains neutral with regard to jurisdictional claims in published maps and institutional affiliations. 